\title{
Supplemental Materials for Regulatory Effectiveness of Social Support
}

\section{Contents}

Using Posterior Probabilities to Assess Between-Person Heterogeneity in Diary Studies (Studies 3-5)

Using Posterior Probabilities to Assess Cardiovascular Reactivity (Study 7)

Using Posterior Probabilities to Assess Heterogeneity due to Studies and Outcomes (Meta-Analysis)

Daily Diary Pilot Study

Lab Support Discussions Pilot Study

Correlations Tables, Studies 2-7

Random Effects Tables, Studies 3-5

Additional Speech Performance Variables, Study 7

Cardiovascular Results Tables, Study 7

Meta-Analysis without Support Effectiveness

Results Summaries for Models with RES and PR Analyzed Separately

References 


\section{Using Posterior Probabilities to Assess Between-Person Heterogeneity in Diary Studies (Studies 3-5)}

To further assess between-person heterogeneity in effects of RES and PR, we capitalized on our Bayesian approach by dividing the posterior distribution for the random effect by the corresponding posterior distribution for its fixed effect. This allowed us to assess the percentage of posterior samples falling above the proposed .25 cutoff (Bolger, Zee, Rossignac-Milon, \& Hassin, 2019). For all effects of RES and PR from across the three diary studies, these posterior probabilities collectively pointed in favor of a relative size of .25 or larger, thereby underscoring the relatively high degree of between-subject heterogeneity in the effects of RES and PR.

\section{Using Posterior Probabilities to Assess Cardiovascular Reactivity (Study 7)}

We also sought to assess patterns of stress reactivity at higher levels of RES and PR across all study phases in a more holistic way, an approach made possible by our use of Bayesian modeling. To do this, we computed the percentage of posterior samples in which all three of the interaction effects involving RES were negative, which would reflect attenuated physiological reactivity during the speech for participants higher than average on RES. We found a 0.67 probability that participants higher (vs. lower) on RES showed tempered IBI reactivity across all three phases. We also repeated this procedure for PR, however there was only a 0.1 probability that participants higher (vs. lower) on PR showed tempered IBI reactivity across all phases. Overall, these results provide preliminary evidence that RES and PR differentially affected patterns of cardiovascular reactivity in response to the stressor. 


\section{Using Posterior Probabilities to Assess Heterogeneity due to Studies and Outcomes (Meta- Analysis)}

To further assess heterogeneity due to studies and outcomes, we capitalized on our Bayesian approach and used a version of the procedure for assessing between-subject heterogeneity in the diary studies (Studies 3-5), as described in this Supplement under Using Posterior Probabilities to Assess Between-Person Heterogeneity in Diary Studies (Studies 3-5). We divided the posterior distribution for the random effect by the corresponding posterior distribution for its fixed effect, thereby creating a posterior distribution for the relative size of the random effect to the fixed effect. This allowed us to assess the percentage of posterior samples falling above the .25 cutoff, a type of posterior probability. Generally, posterior probabilities for the meta-analysis results suggested strong evidence in favor of noteworthy heterogeneity. For meta-analytic effects of RES and PR, nearly all of these posterior draws assessing the degree of heterogeneity due to outcome exceed .25, RES: 0.99; PR: 1 . In contrast, the degree of heterogeneity due to study was somewhat less pronounced, indicated by smaller percentages of these posterior draws exceeding .25, RES: 0.33; PR: 0.75 .

There was a somewhat different pattern for RES-PR (the difference in their effects). Posterior probabilities assessing the percentage of posterior samples exceeding .25 was 0.7 for heterogeneity due to study, and 0.32 for heterogeneity due to outcomes.

Overall, these findings suggested that there was heterogeneity in the predicted effects for RES, PR, and their difference across studies and across outcomes, and that the heterogeneity in effects of RES and PR were largely due to outcomes, whereas heterogeneity in the difference of RES and PR was largely due to studies. 


\section{Daily Diary Pilot Study}

One hundred and six participants participated in a five-night daily diary study. Participants were students enrolled in eligible psychology courses at [masked for review] who received course credit in exchange for their participation. The conclusion of the academic year served as our data collection stopping rule. Participants were 21 years old on average $(S D=4.7)$. There were 68 female participants, 36 male participants, and 2 participants who did not report their gender. The majority of participants completed all five $(n=77)$ or four $(n$ $=16$ ) diary questionnaires on time.

The methods and procedure used in this study were largely the same as those presented for the diary studies in main text. The main difference was that in this pilot study, participants did not nominate a target relationship partner. Instead, they were asked to take into account any support received that day in their ratings of RES and PR. Participants made daily ratings of RES, PR, negative mood, positive mood, and perceptions of support effectiveness using the measures described in the main text; no relational outcomes were measured in this study.

The analysis approach was the same as the approach described in the main text. Fixed effects are displayed in Table S1, differences in effects of RES and PR are displayed in Table S2, and random effects are displayed in Table S3. Within-person and between-person effects of RES and PR and their difference are also shown in Figures S1 and S2, respectively.

Truth facet reliability: Between-person (time nested within-person) reliability $=0.72$, Within-person reliability $=0.86$, and Reliability of change $=0.87$.

Control facet reliability: Between-person (time nested within-person) reliability $=0.71$, Within-person reliability $=0.87$, and Reliability of change $=0.9$.

RES composite reliability: Between-person (time nested within-person) reliability $=0.75$, Within-person reliability $=0.7$, and Reliability of change $=0.74$.

RES-PR correlations: $r_{\text {Within }}=0.38$. $r_{\text {Between }}=0.54$. 


\section{Table S1}

Summary of results from pilot daily diary study, with unstandardized coefficients

\begin{tabular}{llccccccc}
\hline DV & Predictor & Estimate & SE & Lower & Upper & Post_Prob & N_Subj & N_Obs \\
\hline Support Effectiveness & Intercept & 4.75 & 0.09 & 4.57 & 4.94 & - & 100 & 298 \\
Support Effectiveness & RES-within & 0.49 & 0.09 & 0.32 & 0.66 & 1.00 & 100 & 298 \\
Support Effectiveness & PR-within & 0.21 & 0.08 & 0.06 & 0.36 & 1.00 & 100 & 298 \\
Support Effectiveness & RES-between & 0.60 & 0.09 & 0.42 & 0.78 & 1.00 & 100 & 298 \\
Support Effectiveness & PR-between & 0.25 & 0.10 & 0.05 & 0.45 & 0.99 & 100 & 298 \\
Support Effectiveness & Day & 0.03 & 0.04 & -0.05 & 0.10 & - & 100 & 298 \\
\hline Negative Mood & Intercept & 2.97 & 0.12 & 2.73 & 3.21 & - & 100 & 302 \\
Negative Mood & RES-within & -0.13 & 0.07 & -0.27 & 0.02 & 0.96 & 100 & 302 \\
Negative Mood & PR-within & 0.02 & 0.07 & -0.11 & 0.15 & 0.39 & 100 & 302 \\
Negative Mood & RES-between & -0.11 & 0.12 & -0.34 & 0.13 & 0.82 & 100 & 302 \\
Negative Mood & PR-between & -0.14 & 0.13 & -0.40 & 0.13 & 0.85 & 100 & 302 \\
Negative Mood & Day & -0.17 & 0.05 & -0.27 & -0.07 & - & 100 & 302 \\
\hline Positive Mood & Intercept & 3.72 & 0.11 & 3.50 & 3.93 & - & 100 & 302 \\
Positive Mood & RES-within & 0.18 & 0.09 & 0.001 & 0.36 & 0.98 & 100 & 302 \\
Positive Mood & PR-within & 0.05 & 0.07 & -0.10 & 0.19 & 0.74 & 100 & 302 \\
Positive Mood & RES-between & 0.19 & 0.11 & -0.02 & 0.40 & 0.96 & 100 & 302 \\
Positive Mood & PR-between & -0.02 & 0.12 & -0.25 & 0.22 & 0.45 & 100 & 302 \\
Positive Mood & Day & 0.15 & 0.06 & 0.04 & 0.27 & - & 100 & 302 \\
\hline
\end{tabular}

Note. RES = Regulatory Effectiveness of Support. PR = Perceived Responsiveness. Lower and Upper refer to $95 \%$ credibility intervals.

Post_Prob is the posterior probability that the effect is in the hypothesized direction. 


\section{Table S2}

Summary of differences in within-person and between-person effects of RES and PR, pilot daily diary study

\begin{tabular}{llccccc}
\hline Type & DV & RES-PR & SE & Lower & Upper & Post_Prob \\
\hline Within & Support Effectiveness & 0.28 & 0.13 & 0.02 & 0.54 & 0.98 \\
Within & Negative Mood & -0.14 & 0.12 & -0.37 & 0.08 & 0.90 \\
Within & Positive Mood & 0.13 & 0.14 & -0.13 & 0.40 & 0.84 \\
\hline Between & Support Effectiveness & 0.35 & 0.17 & 0.01 & 0.68 & 0.98 \\
Between & Negative Mood & 0.03 & 0.23 & -0.42 & 0.47 & 0.45 \\
Between & Positive Mood & 0.20 & 0.20 & -0.19 & 0.59 & 0.84 \\
\hline
\end{tabular}

Note. RES = Regulatory Effectiveness of Support. PR = Perceived Responsiveness. Type = Type of effect (within-person or betweenperson). Lower and Upper refer to 95\% credibility intervals. Post_Prob is the posterior probability that the effect is in the hypothesized direction. 


\section{Table S3}

Summary of random effects, pilot daily diary study 

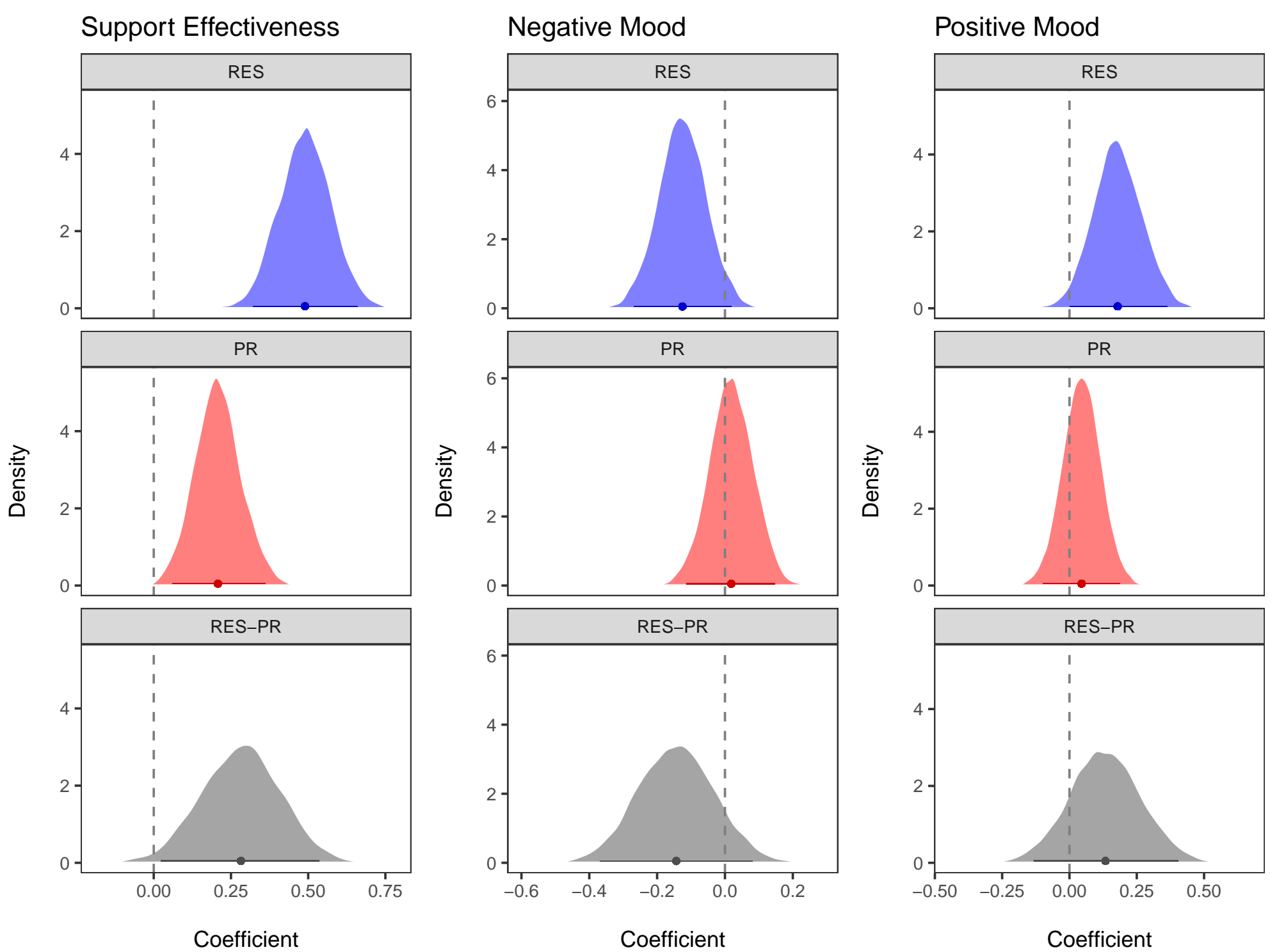

Figure S1. Posterior distributions of within-person effects of regulatory effectiveness of support (RES) and perceived responsiveness (PR) and the difference in their effects (RES-PR), pilot diary study. 

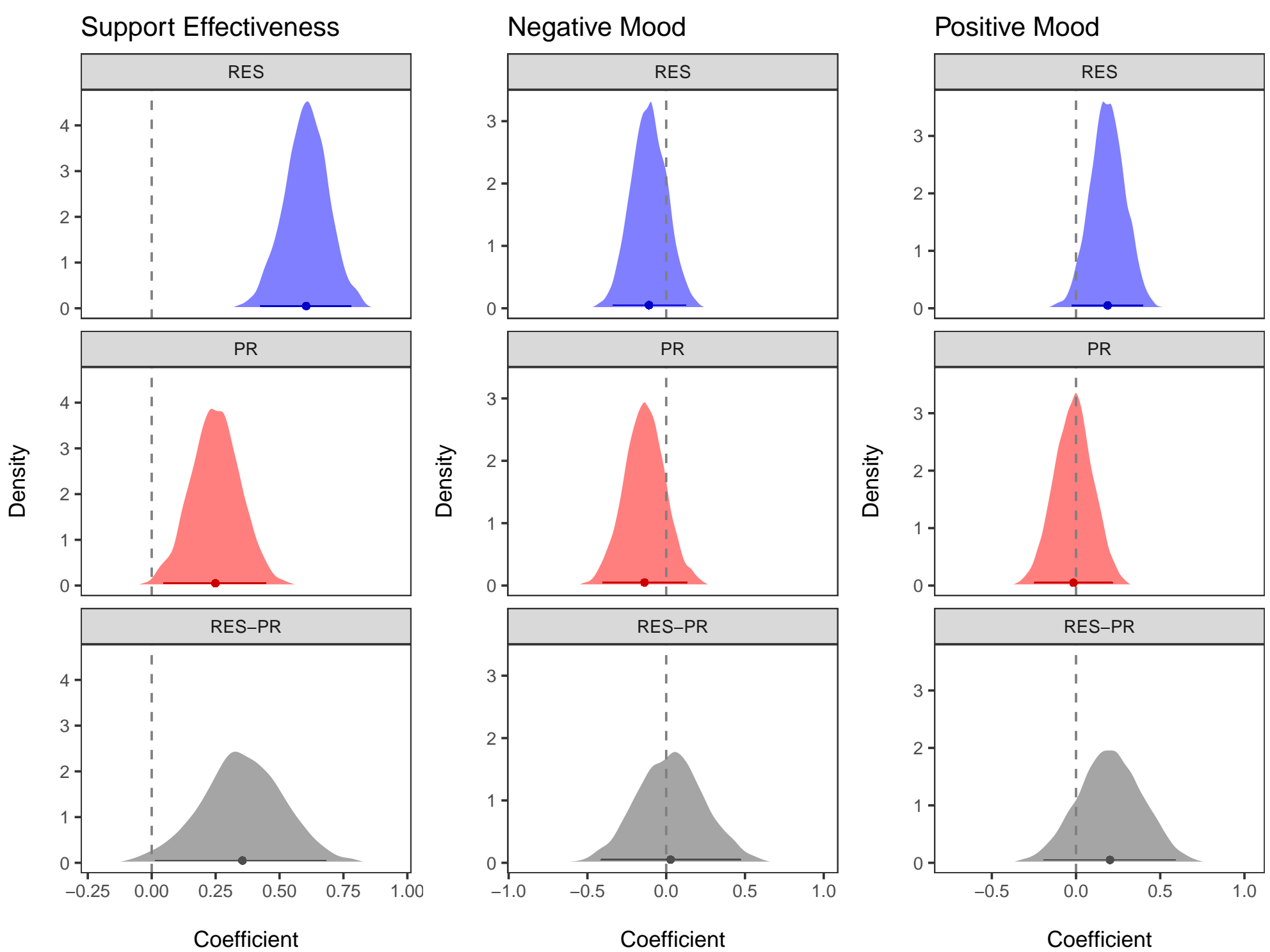

Figure S2. Posterior distributions of between-person effects of regulatory effectiveness of support (RES) and perceived responsiveness (PR) and the difference in their effects (RES-PR), pilot diary study. 


\section{Lab Support Discussions Pilot Study}

This pilot involved secondary analysis of existing data that were collected in connection with separate hypotheses regarding social support discussions. This dataset was previously used in publications by Cavallo, Zee, \& Higgins (2016) and Zee, Cavallo, Flores, Bolger, \& Higgins (2018). With the exception of coder-rated support effectiveness, all dependent measures discussed here were examined in one of these prior publications.

Eighty five friend dyads participated in a laboratory study involving support discussions. Participants were students who received course credit in exchange for their participation or $\$ 5$ cash. Due to equipment issues and failure to follow instructions, data were not available for two dyads. After completing baseline individual difference measures, one partner was randomly assigned to discuss an ongoing issue and their partner was instructed to help in whatever way seemed appropriate. Following a five minute support discussion, participants completed measures regarding their feelings and perceptions of the support discussion; only recipients' ratings were used in the present analyses. Specifically, participants rated their negative mood ( 7 items; $\alpha=0.92)$, positive mood (6 items; $\alpha=0.92)$, feelings of closeness to the provider ( 3 items; $\alpha=0.80)(1=$ Not at all, $7=$ Extremely). Note that mood ratings were only available for 77 dyads, as 6 dyads used check-marks rather than providing ratings along the scale provided. Additional measures were also collected but were not assessed in regards to the present research question. Video data were available for 82 dyads. Two coders blind to hypotheses rated the support discussions for levels of RES (ICC (A, 2) $=0.87)$ and PR $(\operatorname{ICC}(\mathrm{A}, 2)=0.93)$ using versions of the coding schemes described in Study 6 , as well as one item tapping general perceptions of support effectiveness (ICC(A, 2) $=0.76)$. Self-reported measures of RES were not collected in this study.

Results are presented below. The pattern of results was less clear compared to the results obtained in other studies. Although it is unclear why this was the case, there were a few possibilities. First, this study had a somewhat smaller sample size compared to the laboratory studies presented in the main text (Studies 6 and 7). Second, ratings were obtained from two coders. It is possible that using more coders, as we did in Study 6, would help to reduce measurement error. We also obtained a higher correlation between coder-rated RES and PR in this study $(r=0.72)$ compared Study 6. Nevertheless, results are presented here for transparency. 


\section{Table S4}

Summary of results from friend dyad pilot study, with unstandardized coefficients

\begin{tabular}{|c|c|c|c|c|c|c|c|c|}
\hline DV & Predictor & Estimate & $\mathrm{SE}$ & Lower & Upper & Post_Prob & N_Subj & $\mathrm{N} \_\mathrm{Obs}$ \\
\hline Coder-Rated Support Effectiveness & Intercept & 4.38 & 0.08 & 4.22 & 4.53 & - & 82 & 82 \\
\hline Coder-Rated Support Effectiveness & Coder-Rated RES & 0.41 & 0.11 & 0.19 & 0.64 & 1.00 & 82 & 82 \\
\hline Coder-Rated Support Effectiveness & Coder-Rated PR & 0.57 & 0.11 & 0.37 & 0.78 & 1.00 & 82 & 82 \\
\hline Negative Mood & Intercept & 1.78 & 0.14 & 1.50 & 2.06 & - & 77 & 77 \\
\hline Negative Mood & Coder-Rated RES & -0.19 & 0.22 & -0.63 & 0.26 & 0.80 & 77 & 77 \\
\hline Negative Mood & Coder-Rated PR & 0.13 & 0.21 & -0.30 & 0.55 & 0.27 & 77 & 77 \\
\hline Negative Mood & Pre Neg. Mood & 0.11 & 0.11 & -0.11 & 0.33 & - & 77 & 77 \\
\hline Positive Mood & Intercept & 5.04 & 0.16 & 4.72 & 5.36 & - & 77 & 77 \\
\hline Positive Mood & Coder-Rated RES & -0.08 & 0.25 & -0.59 & 0.41 & 0.37 & 77 & 77 \\
\hline Positive Mood & Coder-Rated PR & 0.27 & 0.24 & -0.20 & 0.73 & 0.88 & 77 & 77 \\
\hline Positive Mood & Pre Pos. Mood & 0.07 & 0.12 & -0.17 & 0.30 & - & 77 & 77 \\
\hline Closeness & Intercept & 6.00 & 0.06 & 5.88 & 6.13 & - & 82 & 82 \\
\hline Closeness & Coder-Rated RES & -0.08 & 0.10 & -0.28 & 0.12 & 0.20 & 82 & 82 \\
\hline Closeness & Coder-Rated PR & 0.22 & 0.09 & 0.03 & 0.41 & 0.99 & 82 & 82 \\
\hline Closeness & Pre Closeness & 1.01 & 0.10 & 0.82 & 1.20 & - & 82 & 82 \\
\hline
\end{tabular}

Note. RES = Regulatory Effectiveness of Support. PR = Perceived Responsiveness. Lower and Upper refer to $95 \%$ credibility intervals. Post_Prob is the posterior probability that the effect is in the hypothesized direction. 


\section{Table S5}

Summary of differences in effects of RES and PR, friend dyad pilot study

\begin{tabular}{lccccc}
\hline DV & Term & Estimate & SE & Lower & Upper \\
\hline Support Effectiveness & -0.16 & 0.21 & -0.55 & 0.24 & 0.21 \\
Negative Mood & -0.32 & 0.41 & -1.12 & 0.48 & 0.78 \\
Positive Mood & -0.35 & 0.46 & -1.28 & 0.56 & 0.21 \\
Closeness & -0.31 & 0.18 & -0.66 & 0.04 & 0.95 \\
\hline
\end{tabular}

Note. RES = Regulatory Effectiveness of Support. PR = Perceived Responsiveness. Lower and Upper refer to $95 \%$ credibility intervals. Post_Prob is the posterior probability that the effect is in the hypothesized direction. 

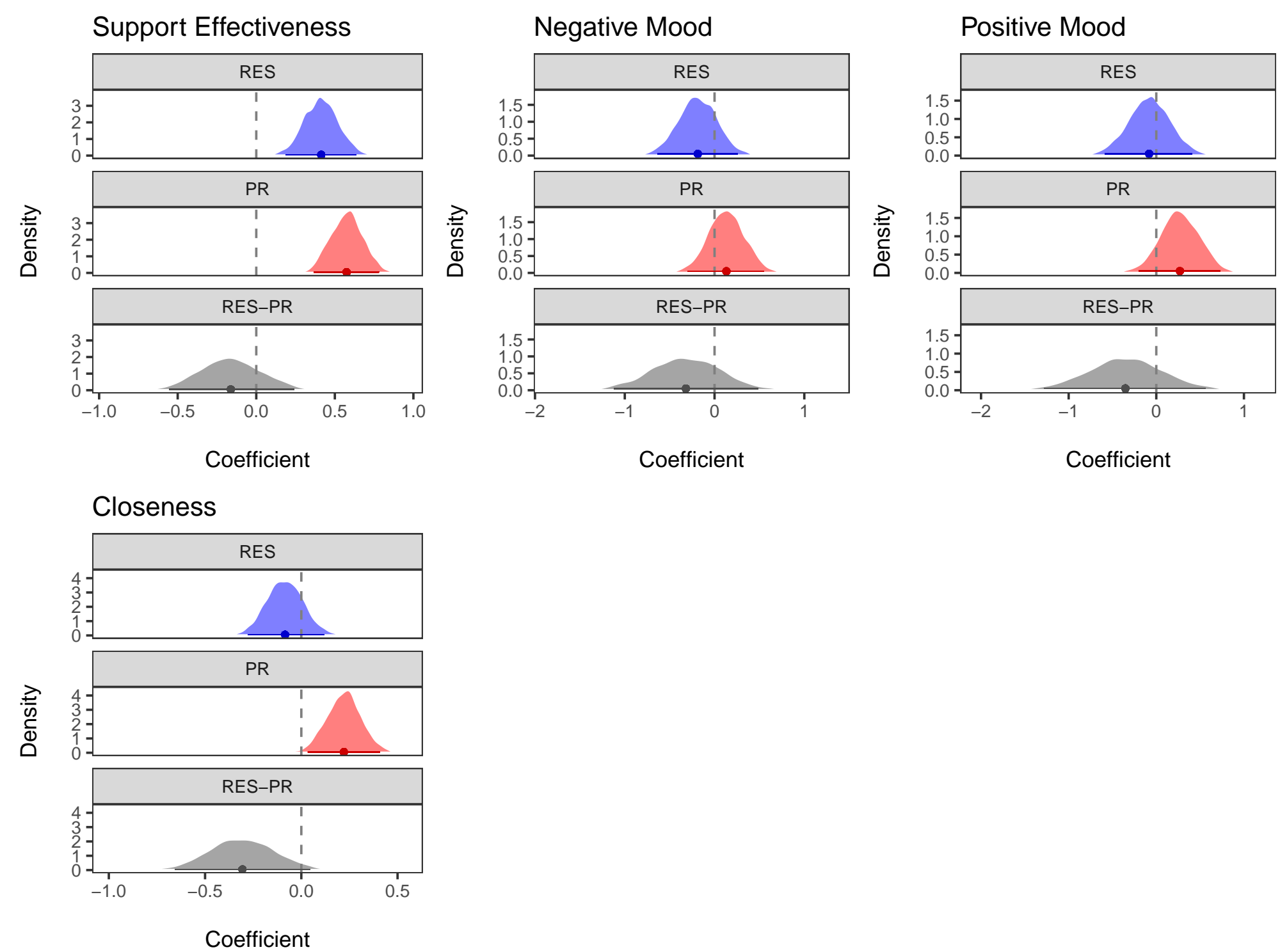

Figure S3. Posterior distributions of effects of coder-rated regulatory effectiveness of support (RES) and coder-rated perceived responsiveness (PR) and the difference in their effects (RES-PR), pilot laboratory study. 


\section{Correlations Tables, Studies 2-7}

\section{Table S6}

Correlations among variables, Study 2

\begin{tabular}{lccc}
\hline Variables & Estimate & Lower & Upper \\
\hline RES, PR & 0.65 & 0.56 & 0.73 \\
RES, Neg. Mood & -0.42 & -0.53 & -0.30 \\
RES, Pos. Mood & 0.55 & 0.45 & 0.64 \\
RES, IOS & 0.41 & 0.29 & 0.53 \\
PR, Neg. Mood & -0.34 & -0.46 & -0.21 \\
PR, Pos. Mood & 0.49 & 0.38 & 0.59 \\
PR, IOS & 0.52 & 0.42 & 0.63 \\
Neg. Mood, Pos. Mood & -0.29 & -0.41 & -0.15 \\
Neg. Mood, IOS & -0.34 & -0.46 & -0.21 \\
Pos. Mood, IOS & 0.43 & 0.31 & 0.54 \\
\hline
\end{tabular}

Note. RES = Regulatory Effectiveness of Support. PR = Perceived Responsiveness. IOS = Inclusion of Other in the Self. 


\section{Table S7A}

Within-person correlations among variables, Studies 3-5

\begin{tabular}{|c|c|c|c|c|c|c|c|c|c|}
\hline \multirow[b]{2}{*}{ Variables } & \multicolumn{3}{|c|}{ Study 3} & \multicolumn{3}{|c|}{ Study 4} & \multicolumn{3}{|c|}{ Study 5} \\
\hline & Estimate & Lower & Upper & Estimate & Lower & Upper & Estimate & Lower & Upper \\
\hline RES, PR & 0.39 & 0.32 & 0.46 & 0.53 & 0.45 & 0.61 & 0.37 & 0.29 & 0.45 \\
\hline RES, Sup. Eff. & 0.41 & 0.34 & 0.48 & 0.45 & 0.37 & 0.53 & 0.38 & 0.30 & 0.46 \\
\hline RES, Neg. Mood & -0.20 & -0.28 & -0.12 & -0.15 & -0.24 & -0.07 & -0.10 & -0.18 & -0.03 \\
\hline RES, Pos. Mood & 0.22 & 0.14 & 0.30 & 0.10 & 0.02 & 0.19 & 0.05 & -0.03 & 0.13 \\
\hline RES, Coping & 0.17 & 0.09 & 0.25 & 0.12 & 0.04 & 0.21 & 0.16 & 0.09 & 0.24 \\
\hline RES, IOS & 0.29 & 0.21 & 0.37 & 0.29 & 0.21 & 0.37 & 0.27 & 0.19 & 0.34 \\
\hline RES, Sleep Quality & - & - & - & 0.07 & -0.03 & 0.17 & -0.05 & -0.15 & 0.05 \\
\hline RES, Task Motivation & - & - & - & - & - & - & 0.07 & -0.03 & 0.17 \\
\hline RES, Task Performance & - & - & - & - & - & - & 0.04 & -0.04 & 0.13 \\
\hline PR, Sup. Eff. & 0.36 & 0.28 & 0.43 & 0.41 & 0.32 & 0.49 & 0.34 & 0.25 & 0.43 \\
\hline PR, Neg. Mood & -0.15 & -0.23 & -0.07 & -0.10 & -0.19 & -0.02 & -0.06 & -0.14 & 0.03 \\
\hline PR, Pos. Mood & 0.19 & 0.11 & 0.27 & 0.12 & 0.04 & 0.20 & 0.10 & 0.02 & 0.18 \\
\hline PR, Coping & 0.10 & 0.02 & 0.18 & 0.10 & 0.01 & 0.19 & 0.25 & 0.16 & 0.33 \\
\hline $\mathrm{PR}, \mathrm{IOS}$ & 0.42 & 0.35 & 0.48 & 0.40 & 0.32 & 0.48 & 0.33 & 0.25 & 0.41 \\
\hline PR, Sleep Quality & - & - & - & 0.02 & -0.09 & 0.12 & 0.03 & -0.07 & 0.13 \\
\hline PR, Task Motivation & - & - & - & - & - & - & 0.09 & -0.02 & 0.19 \\
\hline PR, Task Performance & - & - & - & - & - & - & 0.03 & -0.06 & 0.13 \\
\hline Sup. Eff., Neg. Mood & -0.13 & -0.21 & -0.05 & -0.10 & -0.18 & -0.02 & -0.10 & -0.17 & -0.02 \\
\hline Sup. Eff., Pos. Mood & 0.19 & 0.11 & 0.27 & 0.06 & -0.03 & 0.14 & 0.02 & -0.05 & 0.10 \\
\hline Sup. Eff., Coping & 0.17 & 0.09 & 0.25 & 0.15 & 0.06 & 0.23 & 0.12 & 0.04 & 0.20 \\
\hline Sup. Eff., IOS & 0.23 & 0.15 & 0.30 & 0.35 & 0.27 & 0.43 & 0.24 & 0.16 & 0.32 \\
\hline Sup. Eff., Sleep Quality & - & - & - & -0.02 & -0.12 & 0.07 & -0.03 & -0.12 & 0.07 \\
\hline Sup. Eff., Task Motivation & - & - & - & - & - & - & -0.02 & -0.12 & 0.07 \\
\hline Sup. Eff., Task Performance & - & - & - & - & - & - & -0.03 & -0.12 & 0.06 \\
\hline
\end{tabular}

Note. RES = Regulatory Effectiveness of Support. PR = Perceived Responsiveness. IOS = Inclusion of Other in the Self. Sup. Eff. = Support Effectiveness. 


\section{Table S7B}

Within-person correlations among variables, Studies 3-5, continued

\begin{tabular}{|c|c|c|c|c|c|c|c|c|c|}
\hline \multirow[b]{2}{*}{ Variables } & \multicolumn{3}{|c|}{ Study 3} & \multicolumn{3}{|c|}{ Study 4} & \multicolumn{3}{|c|}{ Study 5} \\
\hline & Estimate & Lower & Upper & Estimate & Lower & Upper & Estimate & Lower & Upper \\
\hline Neg. Mood, Pos. Mood & -0.47 & -0.54 & -0.41 & -0.50 & -0.56 & -0.44 & -0.48 & -0.54 & -0.43 \\
\hline Neg. Mood, Coping & -0.01 & -0.09 & 0.08 & 0.10 & 0.03 & 0.17 & 0.04 & -0.02 & 0.10 \\
\hline Neg. Mood, IOS & -0.21 & -0.29 & -0.13 & -0.11 & -0.18 & -0.04 & -0.12 & -0.19 & -0.06 \\
\hline Neg. Mood, Sleep Quality & - & - & - & -0.01 & -0.10 & 0.07 & -0.09 & -0.17 & -0.01 \\
\hline Neg. Mood, Task Motivation & - & - & - & - & - & - & -0.13 & -0.21 & -0.06 \\
\hline Neg. Mood, Task Performance & - & - & - & - & - & - & -0.10 & -0.17 & -0.02 \\
\hline Pos. Mood, Coping & 0.10 & 0.02 & 0.19 & 0.02 & -0.05 & 0.09 & 0.07 & 0.01 & 0.14 \\
\hline Pos. Mood, IOS & 0.18 & 0.09 & 0.26 & 0.21 & 0.13 & 0.27 & 0.16 & 0.10 & 0.22 \\
\hline Pos. Mood, Sleep Quality & - & - & - & 0.07 & -0.01 & 0.15 & 0.05 & -0.03 & 0.13 \\
\hline Pos. Mood, Task Motivation & - & - & - & - & - & - & 0.14 & 0.06 & 0.22 \\
\hline Pos. Mood, Task Performance & - & - & - & - & - & - & 0.13 & 0.05 & 0.20 \\
\hline Coping, IOS & 0.10 & 0.01 & 0.18 & 0.27 & 0.20 & 0.34 & 0.23 & 0.17 & 0.30 \\
\hline Coping, Sleep Quality & - & - & - & 0.04 & -0.04 & 0.12 & -0.07 & -0.15 & 0.01 \\
\hline Coping, Task Motivation & - & - & - & - & - & - & -0.01 & -0.09 & 0.07 \\
\hline Coping, Task Performance & - & - & - & - & - & - & 0.03 & -0.04 & 0.11 \\
\hline IOS, Sleep Quality & - & - & - & 0.04 & -0.05 & 0.12 & 0.00 & -0.08 & 0.08 \\
\hline IOS, Task Motivation & - & - & - & - & - & - & 0.00 & -0.08 & 0.08 \\
\hline IOS, Task Performance & - & - & - & - & - & - & 0.03 & -0.05 & 0.11 \\
\hline Sleep Quality, Task Motivation & - & - & - & - & - & - & 0.07 & -0.02 & 0.16 \\
\hline Sleep Quality, Task Performance & - & - & - & - & - & - & 0.16 & 0.08 & 0.25 \\
\hline Task Motivation, Task Performance & - & - & - & - & - & - & 0.33 & 0.25 & 0.41 \\
\hline
\end{tabular}

Note. $\mathrm{RES}=$ Regulatory Effectiveness of Support. PR = Perceived Responsiveness. IOS = Inclusion of Other in the Self. Sup. Eff. $=$ Support Effectiveness. 


\section{Table S8A}

Between-person correlations among variables, Studies 3-5

\begin{tabular}{|c|c|c|c|c|c|c|c|c|c|}
\hline \multirow[b]{2}{*}{ Variables } & \multicolumn{3}{|c|}{ Study 3} & \multicolumn{3}{|c|}{ Study 4} & \multicolumn{3}{|c|}{ Study 5} \\
\hline & Estimate & Lower & Upper & Estimate & Lower & Upper & Estimate & Lower & Upper \\
\hline RES, PR & 0.64 & 0.55 & 0.72 & 0.66 & 0.56 & 0.74 & 0.54 & 0.43 & 0.63 \\
\hline RES, Sup. Eff. & 0.48 & 0.35 & 0.58 & 0.63 & 0.52 & 0.72 & 0.48 & 0.36 & 0.59 \\
\hline RES, Neg. Mood & -0.15 & -0.29 & -0.01 & -0.31 & -0.45 & -0.16 & -0.15 & -0.28 & 0.00 \\
\hline RES, Pos. Mood & 0.26 & 0.12 & 0.40 & 0.39 & 0.24 & 0.51 & 0.36 & 0.23 & 0.48 \\
\hline RES, Coping & 0.17 & 0.03 & 0.30 & 0.22 & 0.06 & 0.36 & 0.05 & -0.09 & 0.20 \\
\hline RES, IOS & 0.31 & 0.17 & 0.44 & 0.51 & 0.38 & 0.62 & 0.28 & 0.14 & 0.41 \\
\hline RES, Sleep Quality & - & - & - & 0.19 & 0.03 & 0.34 & 0.10 & -0.04 & 0.24 \\
\hline RES, Task Motivation & - & - & - & - & - & - & 0.20 & 0.06 & 0.33 \\
\hline RES, Task Performance & - & - & - & - & - & - & 0.20 & 0.06 & 0.34 \\
\hline PR, Sup. Eff. & 0.52 & 0.40 & 0.61 & 0.61 & 0.49 & 0.71 & 0.50 & 0.38 & 0.61 \\
\hline PR, Neg. Mood & -0.13 & -0.27 & 0.01 & -0.22 & -0.37 & -0.06 & -0.18 & -0.31 & -0.04 \\
\hline PR, Pos. Mood & 0.11 & -0.04 & 0.25 & 0.17 & 0.02 & 0.32 & 0.21 & 0.07 & 0.34 \\
\hline PR, Coping & 0.20 & 0.06 & 0.34 & 0.21 & 0.05 & 0.36 & 0.03 & -0.12 & 0.17 \\
\hline $\mathrm{PR}, \mathrm{IOS}$ & 0.50 & 0.39 & 0.60 & 0.59 & 0.47 & 0.69 & 0.48 & 0.36 & 0.58 \\
\hline PR, Sleep Quality & - & - & - & 0.10 & -0.06 & 0.26 & 0.23 & 0.09 & 0.36 \\
\hline PR, Task Motivation & - & - & - & - & - & - & 0.09 & -0.06 & 0.23 \\
\hline PR, Task Performance & - & - & - & - & - & - & 0.14 & -0.01 & 0.27 \\
\hline Sup. Eff., Neg. Mood & -0.13 & -0.27 & 0.02 & -0.19 & -0.34 & -0.03 & -0.21 & -0.35 & -0.07 \\
\hline Sup. Eff., Pos. Mood & 0.11 & -0.04 & 0.25 & 0.24 & 0.09 & 0.39 & 0.21 & 0.07 & 0.34 \\
\hline Sup. Eff., Coping & 0.15 & 0.00 & 0.29 & 0.18 & 0.02 & 0.33 & 0.02 & -0.12 & 0.17 \\
\hline Sup. Eff., IOS & 0.29 & 0.16 & 0.42 & 0.52 & 0.39 & 0.63 & 0.24 & 0.10 & 0.38 \\
\hline Sup. Eff., Sleep Quality & - & - & - & 0.16 & 0.01 & 0.31 & 0.14 & -0.01 & 0.28 \\
\hline Sup. Eff., Task Motivation & - & - & - & - & - & - & 0.16 & 0.03 & 0.30 \\
\hline Sup. Eff., Task Performance & - & - & - & - & - & - & 0.20 & 0.06 & 0.34 \\
\hline
\end{tabular}

Note. RES = Regulatory Effectiveness of Support. PR = Perceived Responsiveness. IOS = Inclusion of Other in the Self. Sup. Eff. = Support Effectiveness. 


\section{Table S8B}

Between-person correlations among variables, Studies 3-5, continued

\begin{tabular}{|c|c|c|c|c|c|c|c|c|c|}
\hline \multirow[b]{2}{*}{ Variables } & \multicolumn{3}{|c|}{ Study 3} & \multicolumn{3}{|c|}{ Study 4} & \multicolumn{3}{|c|}{ Study 5} \\
\hline & Estimate & Lower & Upper & Estimate & Lower & Upper & Estimate & Lower & Upper \\
\hline Neg. Mood, Pos. Mood & -0.30 & -0.43 & -0.16 & -0.41 & -0.54 & -0.26 & -0.16 & -0.31 & -0.02 \\
\hline Neg. Mood, Coping & -0.06 & -0.21 & 0.08 & 0.04 & -0.12 & 0.21 & 0.10 & -0.05 & 0.24 \\
\hline Neg. Mood, IOS & -0.16 & -0.29 & -0.02 & -0.22 & -0.37 & -0.05 & -0.14 & -0.28 & 0.00 \\
\hline Neg. Mood, Sleep Quality & - & - & - & -0.15 & -0.31 & 0.01 & -0.33 & -0.45 & -0.20 \\
\hline Neg. Mood, Task Motivation & - & - & - & - & - & - & -0.02 & -0.16 & 0.13 \\
\hline Neg. Mood, Task Performance & - & - & - & - & - & - & -0.09 & -0.23 & 0.05 \\
\hline Pos. Mood, Coping & 0.13 & -0.01 & 0.27 & -0.10 & -0.26 & 0.06 & 0.11 & -0.04 & 0.25 \\
\hline Pos. Mood, IOS & 0.20 & 0.06 & 0.34 & 0.28 & 0.13 & 0.42 & 0.15 & 0.00 & 0.29 \\
\hline Pos. Mood, Sleep Quality & - & - & - & 0.28 & 0.13 & 0.42 & 0.30 & 0.16 & 0.43 \\
\hline Pos. Mood, Task Motivation & - & - & - & - & - & - & 0.08 & -0.07 & 0.22 \\
\hline Pos. Mood, Task Performance & - & - & - & - & - & - & 0.11 & -0.04 & 0.25 \\
\hline Coping, IOS & 0.11 & -0.03 & 0.25 & 0.15 & -0.01 & 0.31 & -0.05 & -0.20 & 0.10 \\
\hline Coping, Sleep Quality & - & - & - & -0.03 & -0.18 & 0.14 & 0.04 & -0.11 & 0.18 \\
\hline Coping, Task Motivation & - & - & - & - & - & - & 0.04 & -0.10 & 0.19 \\
\hline Coping, Task Performance & - & - & - & - & - & - & -0.11 & -0.25 & 0.03 \\
\hline IOS, Sleep Quality & - & - & - & 0.23 & 0.07 & 0.37 & 0.07 & -0.08 & 0.21 \\
\hline IOS, Task Motivation & - & - & - & - & - & - & 0.02 & -0.13 & 0.16 \\
\hline IOS, Task Performance & - & - & - & - & - & - & 0.06 & -0.09 & 0.21 \\
\hline Sleep Quality, Task Motivation & - & - & - & - & - & - & 0.13 & -0.01 & 0.27 \\
\hline Sleep Quality, Task Performance & - & - & - & - & - & - & 0.11 & -0.03 & 0.25 \\
\hline Task Motivation, Task Performance & - & - & - & - & - & - & 0.49 & 0.37 & 0.60 \\
\hline
\end{tabular}

Note. RES $=$ Regulatory Effectiveness of Support. PR = Perceived Responsiveness. IOS = Inclusion of Other in the Self. Sup. Eff. $=$ Support Effectiveness. 


\section{Table S9A}

Correlations among variables, Study 6

\begin{tabular}{lccc}
\hline Variables & Estimate & Lower & Upper \\
\hline RES, PR & 0.50 & 0.39 & 0.60 \\
RES, Sup. Eff. & 0.73 & 0.66 & 0.79 \\
RES, Neg. Mood & -0.26 & -0.39 & -0.14 \\
RES, Pos. Mood & 0.43 & 0.32 & 0.54 \\
RES, IOS & 0.40 & 0.28 & 0.52 \\
RES, Closeness & 0.40 & 0.27 & 0.51 \\
RES, Coder-Rated RES & 0.25 & 0.12 & 0.37 \\
RES, Coder-Rated PR & 0.11 & -0.02 & 0.24 \\
PR, Sup. Eff. & 0.52 & 0.42 & 0.62 \\
PR, Neg. Mood & -0.15 & -0.28 & -0.01 \\
PR, Pos. Mood & 0.31 & 0.19 & 0.43 \\
PR, IOS & 0.45 & 0.34 & 0.56 \\
PR, Closeness & 0.56 & 0.45 & 0.65 \\
PR, Coder-Rated RES & 0.18 & 0.05 & 0.31 \\
PR, Coder-Rated PR & 0.30 & 0.17 & 0.42 \\
Sup. Eff., Neg. Mood & -0.23 & -0.36 & -0.10 \\
Sup. Eff., Pos. Mood & 0.34 & 0.21 & 0.45 \\
Sup. Eff., IOS & 0.50 & 0.39 & 0.60 \\
Sup. Eff., Closeness & 0.38 & 0.26 & 0.49 \\
Sup. Eff., Coder-Rated RES & 0.27 & 0.14 & 0.39 \\
Sup. Eff., Coder-Rated PR & 0.10 & -0.03 & 0.23 \\
\hline
\end{tabular}

Note. RES $=$ Regulatory Effectiveness of Support. PR $=$ Perceived Responsiveness. IOS $=$ Inclusion of Other in the Self. 


\section{Table S9B}

Correlations among variables, Study 6, continued

\begin{tabular}{lccc}
\hline Variables & Estimate & Lower & Upper \\
\hline Neg. Mood, Pos. Mood & -0.49 & -0.59 & -0.38 \\
Neg. Mood, IOS & -0.25 & -0.38 & -0.12 \\
Neg. Mood, Closeness & -0.20 & -0.32 & -0.06 \\
Neg. Mood, Coder-Rated RES & -0.25 & -0.38 & -0.12 \\
Neg. Mood, Coder-Rated PR & -0.11 & -0.24 & 0.03 \\
Pos. Mood, IOS & 0.31 & 0.18 & 0.43 \\
Pos. Mood, Closeness & 0.22 & 0.09 & 0.35 \\
Pos. Mood, Coder-Rated RES & 0.11 & -0.03 & 0.24 \\
Pos. Mood, Coder-Rated PR & 0.09 & -0.05 & 0.22 \\
IOS, Closeness & 0.40 & 0.27 & 0.51 \\
IOS, Coder-Rated RES & 0.13 & -0.01 & 0.26 \\
IOS, Coder-Rated PR & 0.16 & 0.02 & 0.30 \\
Closeness, Coder-Rated RES & 0.10 & -0.04 & 0.23 \\
Closeness, Coder-Rated PR & 0.18 & 0.05 & 0.31 \\
Coder-Rated RES, Coder-Rated PR & 0.28 & 0.15 & 0.40 \\
\hline
\end{tabular}

Note. RES = Regulatory Effectiveness of Support. PR = Perceived Responsiveness. IOS = Inclusion of Other in the Self. 


\section{Table S10A}

Correlations among variables, Study 7

\begin{tabular}{|c|c|c|c|}
\hline Variables & Estimate & Lower & Upper \\
\hline RES, PR & 0.51 & 0.36 & 0.64 \\
\hline RES, Sup. Eff. & 0.69 & 0.58 & 0.78 \\
\hline RES, Neg. Mood & -0.10 & -0.28 & 0.08 \\
\hline RES, Pos. Mood & 0.03 & -0.15 & 0.21 \\
\hline RES, IOS & 0.34 & 0.18 & 0.50 \\
\hline RES, Closeness & 0.33 & 0.16 & 0.49 \\
\hline RES, Change Motive & 0.11 & -0.08 & 0.28 \\
\hline RES, Help with Speech & 0.51 & 0.36 & 0.64 \\
\hline RES, Performance & -0.08 & -0.26 & 0.11 \\
\hline PR, Sup. Eff. & 0.47 & 0.32 & 0.61 \\
\hline PR, Neg. Mood & -0.25 & -0.42 & -0.07 \\
\hline PR, Pos. Mood & 0.04 & -0.14 & 0.22 \\
\hline $\mathrm{PR}, \mathrm{IOS}$ & 0.40 & 0.23 & 0.55 \\
\hline PR, Closeness & 0.40 & 0.23 & 0.55 \\
\hline PR, Change Motive & 0.00 & -0.18 & 0.18 \\
\hline PR, Help with Speech & 0.26 & 0.09 & 0.43 \\
\hline PR, Performance & -0.20 & -0.37 & -0.02 \\
\hline Sup. Eff., Neg. Mood & -0.13 & -0.31 & 0.06 \\
\hline Sup. Eff., Pos. Mood & 0.11 & -0.07 & 0.29 \\
\hline Sup. Eff., IOS & 0.31 & 0.13 & 0.47 \\
\hline Sup. Eff., Closeness & 0.34 & 0.16 & 0.49 \\
\hline Sup. Eff., Change Motive & 0.10 & -0.09 & 0.28 \\
\hline Sup. Eff., Help with Speech & 0.54 & 0.40 & 0.66 \\
\hline Sup. Eff., Performance & -0.04 & -0.22 & 0.15 \\
\hline
\end{tabular}

Note. RES = Regulatory Effectiveness of Support. PR = Perceived Responsiveness. IOS = Inclusion of Other in the Self. 


\section{Table S10B}

Correlations among variables, Study 7, continued

\begin{tabular}{lccc}
\hline Variables & Estimate & Lower & Upper \\
\hline Neg. Mood, Pos. Mood & -0.10 & -0.27 & 0.09 \\
Neg. Mood, IOS & -0.11 & -0.29 & 0.08 \\
Neg. Mood, Closeness & -0.07 & -0.26 & 0.13 \\
Neg. Mood, Change Motive & -0.03 & -0.22 & 0.17 \\
Neg. Mood, Help with Speech & -0.12 & -0.30 & 0.07 \\
Neg. Mood, Performance & 0.01 & -0.19 & 0.20 \\
Pos. Mood, IOS & -0.08 & -0.27 & 0.12 \\
Pos. Mood, Closeness & 0.09 & -0.10 & 0.28 \\
Pos. Mood, Change Motive & -0.08 & -0.28 & 0.11 \\
Pos. Mood, Help with Speech & 0.16 & -0.03 & 0.33 \\
Pos. Mood, Performance & 0.06 & -0.14 & 0.25 \\
IOS, Closeness & 0.29 & 0.11 & 0.46 \\
IOS, Change Motive & 0.00 & -0.19 & 0.18 \\
IOS, Help with Speech & 0.13 & -0.05 & 0.31 \\
IOS, Performance & -0.05 & -0.24 & 0.14 \\
Closeness, Change Motive & -0.07 & -0.27 & 0.13 \\
Closeness, Help with Speech & 0.35 & 0.18 & 0.51 \\
Change Motive, Help with Speech & 0.12 & -0.07 & 0.30 \\
Closeness, Performance & -0.14 & -0.31 & 0.06 \\
Change Motive, Performance & 0.14 & -0.05 & 0.32 \\
Help with Speech, Performance & 0.10 & -0.08 & 0.28 \\
\hline
\end{tabular}

Note. RES = Regulatory Effectiveness of Support. PR = Perceived Responsiveness. IOS = Inclusion of Other in the Self. 


\section{Random Effects Tables, Studies 3-5}

\section{Table S11A}

Summary of random effects, Study 3

\begin{tabular}{llcccc}
\hline DV & Term & Estimate & SE & Lower & Upper \\
\hline Support Effectiveness & Intercept SD & 0.37 & 0.16 & 0.04 & 0.64 \\
Support Effectiveness & RES SD & 0.15 & 0.10 & 0.007 & 0.35 \\
Support Effectiveness & PR SD & 0.18 & 0.10 & 0.01 & 0.37 \\
Support Effectiveness & Intercept-RES Cor & -0.07 & 0.44 & -0.85 & 0.80 \\
Support Effectiveness & Intercept-PR Cor & -0.21 & 0.44 & -0.90 & 0.74 \\
Support Effectiveness & RES-PR Cor & -0.11 & 0.48 & -0.88 & 0.82 \\
Support Effectiveness & Residual & 1.17 & 0.05 & 1.08 & 1.26 \\
Support Effectiveness & AR(1) & 0.21 & 0.08 & 0.04 & 0.36 \\
\hline Negative Mood & Intercept SD & 0.90 & 0.08 & 0.75 & 1.05 \\
Negative Mood & RES SD & 0.17 & 0.07 & 0.03 & 0.32 \\
Negative Mood & PR SD & 0.19 & 0.10 & 0.01 & 0.40 \\
Negative Mood & Intercept-RES Cor & -0.52 & 0.27 & -0.94 & 0.08 \\
Negative Mood & Intercept-PR Cor & 0.28 & 0.34 & -0.50 & 0.86 \\
Negative Mood & RES-PR Cor & -0.27 & 0.42 & -0.91 & 0.69 \\
Negative Mood & Residual & 1.03 & 0.04 & 0.94 & 1.12 \\
Negative Mood & AR(1) & 0.13 & 0.08 & -0.01 & 0.29 \\
\hline Positive Mood & Intercept SD & 0.63 & 0.16 & 0.18 & 0.86 \\
Positive Mood & RES SD & 0.10 & 0.07 & 0.005 & 0.26 \\
Positive Mood & PR SD & 0.16 & 0.10 & 0.009 & 0.36 \\
Positive Mood & Intercept-RES Cor & -0.24 & 0.44 & -0.91 & 0.71 \\
Positive Mood & Intercept-PR Cor & -0.13 & 0.40 & -0.83 & 0.72 \\
Positive Mood & RES-PR Cor & -0.05 & 0.49 & -0.89 & 0.85 \\
Positive Mood & Residual & 1.18 & 0.06 & 1.08 & 1.30 \\
Positive Mood & AR(1) & 0.20 & 0.10 & 0.009 & 0.41 \\
\hline
\end{tabular}

Note. RES = Regulatory Effectiveness of Support. PR = Perceived Responsiveness. IOS $=$ Inclusion of the other in the self. SD $=$ standard deviation. Cor $=$ correlation. 
Table S11B

Summary of random effects, Study 3, continued

\begin{tabular}{llcccc}
\hline DV & Term & Estimate & SE & Lower & Upper \\
\hline Coping & Intercept SD & 0.49 & 0.15 & 0.10 & 0.71 \\
Coping & RES SD & 0.12 & 0.06 & 0.008 & 0.24 \\
Coping & PR SD & 0.06 & 0.05 & 0.003 & 0.17 \\
Coping & Intercept-RES Cor & -0.08 & 0.37 & -0.79 & 0.71 \\
Coping & Intercept-PR Cor & 0.04 & 0.45 & -0.83 & 0.85 \\
Coping & RES-PR Cor & -0.02 & 0.49 & -0.88 & 0.86 \\
Coping & Residual & 0.84 & 0.05 & 0.75 & 0.93 \\
Coping & AR(1) & 0.38 & 0.13 & 0.11 & 0.61 \\
\hline IOS & Intercept SD & 1.15 & 0.07 & 1.02 & 1.30 \\
IOS & RES SD & 0.18 & 0.07 & 0.02 & 0.31 \\
IOS & PR SD & 0.14 & 0.08 & 0.01 & 0.29 \\
IOS & Intercept-RES Cor & -0.10 & 0.26 & -0.62 & 0.44 \\
IOS & Intercept-PR Cor & -0.06 & 0.34 & -0.71 & 0.65 \\
IOS & RES-PR Cor & 0.28 & 0.45 & -0.69 & 0.93 \\
IOS & Residual & 0.89 & 0.04 & 0.81 & 0.97 \\
IOS & AR(1) & 0.07 & 0.08 & -0.08 & 0.23 \\
\hline
\end{tabular}

Note. RES = Regulatory Effectiveness of Support. $\mathrm{PR}=$ Perceived Responsiveness. IOS $=$ Inclusion of the other in the self. SD $=$ standard deviation. Cor $=$ correlation. 


\section{Table S12A}

Summary of random effects, Study 4

\begin{tabular}{llcccc}
\hline DV & Term & Estimate & SE & Lower & Upper \\
\hline Support Effectiveness & Intercept SD & 0.55 & 0.12 & 0.26 & 0.75 \\
Support Effectiveness & RES SD & 0.27 & 0.12 & 0.04 & 0.51 \\
Support Effectiveness & PR SD & 0.17 & 0.12 & 0.007 & 0.43 \\
Support Effectiveness & Intercept-RES Cor & -0.52 & 0.30 & -0.94 & 0.20 \\
Support Effectiveness & Intercept-PR Cor & 0.07 & 0.44 & -0.80 & 0.84 \\
Support Effectiveness & RES-PR Cor & -0.25 & 0.47 & -0.93 & 0.77 \\
Support Effectiveness & Residual & 1.10 & 0.06 & 0.99 & 1.21 \\
Support Effectiveness & AR(1) & 0.03 & 0.10 & -0.16 & 0.23 \\
\hline Negative Mood & Intercept SD & 0.99 & 0.08 & 0.83 & 1.15 \\
Negative Mood & RES SD & 0.19 & 0.07 & 0.04 & 0.34 \\
Negative Mood & PR SD & 0.13 & 0.09 & 0.006 & 0.34 \\
Negative Mood & Intercept-RES Cor & -0.65 & 0.25 & -0.97 & -0.03 \\
Negative Mood & Intercept-PR Cor & 0.04 & 0.42 & -0.77 & 0.84 \\
Negative Mood & RES-PR Cor & -0.18 & 0.48 & -0.90 & 0.79 \\
Negative Mood & Residual & 1.01 & 0.05 & 0.92 & 1.11 \\
Negative Mood & AR(1) & -0.04 & 0.09 & -0.21 & 0.15 \\
\hline Positive Mood & Intercept SD & 0.78 & 0.07 & 0.65 & 0.92 \\
Positive Mood & RES SD & 0.10 & 0.08 & 0.005 & 0.28 \\
Positive Mood & PR SD & 0.14 & 0.09 & 0.005 & 0.35 \\
Positive Mood & Intercept-RES Cor & -0.07 & 0.45 & -0.85 & 0.81 \\
Positive Mood & Intercept-PR Cor & 0.22 & 0.40 & -0.68 & 0.87 \\
Positive Mood & RES-PR Cor & -0.15 & 0.50 & -0.93 & 0.82 \\
Positive Mood & Residual & 1.09 & 0.05 & 1.00 & 1.19 \\
Positive Mood & AR(1) & -0.25 & 0.08 & -0.40 & -0.10 \\
\hline
\end{tabular}

Note. RES = Regulatory Effectiveness of Support. $\mathrm{PR}=$ Perceived Responsiveness. IOS $=$ Inclusion of the other in the self. $\mathrm{SD}=$ standard deviation. Cor $=$ correlation . 


\section{Table S12B}

Summary of random effects, Study 4, continued

\begin{tabular}{llcccc}
\hline DV & Term & Estimate & SE & Lower & Upper \\
\hline Coping & Intercept SD & 0.76 & 0.06 & 0.63 & 0.88 \\
Coping & RES SD & 0.13 & 0.08 & 0.007 & 0.30 \\
Coping & PR SD & 0.15 & 0.08 & 0.009 & 0.32 \\
Coping & Intercept-RES Cor & -0.19 & 0.37 & -0.86 & 0.60 \\
Coping & Intercept-PR Cor & -0.28 & 0.34 & -0.84 & 0.55 \\
Coping & RES-PR Cor & 0.09 & 0.47 & -0.81 & 0.90 \\
Coping & Residual & 0.74 & 0.04 & 0.67 & 0.82 \\
Coping & AR(1) & 0.06 & 0.10 & -0.12 & 0.26 \\
\hline IOS & Intercept SD & 1.08 & 0.08 & 0.93 & 1.24 \\
IOS & RES SD & 0.24 & 0.09 & 0.03 & 0.40 \\
IOS & PR SD & 0.11 & 0.08 & 0.005 & 0.29 \\
IOS & Intercept-RES Cor & 0.07 & 0.26 & -0.45 & 0.58 \\
IOS & Intercept-PR Cor & 0.13 & 0.41 & -0.74 & 0.86 \\
IOS & RES-PR Cor & 0.04 & 0.47 & -0.82 & 0.87 \\
IOS & Residual & 0.84 & 0.04 & 0.76 & 0.93 \\
IOS & AR(1) & 0.06 & 0.10 & -0.12 & 0.27 \\
\hline Sleep Quality & Intercept SD & 0.97 & 0.11 & 0.75 & 1.17 \\
Sleep Quality & RES SD & 0.15 & 0.10 & 0.006 & 0.38 \\
Sleep Quality & PR SD & 0.18 & 0.12 & 0.008 & 0.43 \\
Sleep Quality & Intercept-RES Cor & 0.09 & 0.44 & -0.78 & 0.86 \\
Sleep Quality & Intercept-PR Cor & 0.38 & 0.40 & -0.61 & 0.93 \\
Sleep Quality & RES-PR Cor & -0.12 & 0.50 & -0.90 & 0.84 \\
Sleep Quality & Residual & 1.02 & 0.08 & 0.87 & 1.19 \\
Sleep Quality & AR(1) & -0.22 & 0.15 & -0.48 & 0.11 \\
\hline
\end{tabular}

Note. RES = Regulatory Effectiveness of Support. PR = Perceived Responsiveness. IOS $=$ Inclusion of the other in the self. $\mathrm{SD}=$ standard deviation. Cor $=$ correlation. 


\section{Table S13A}

Summary of random effects, Study 5

\begin{tabular}{llcccc}
\hline dv & term & Estimate & SE & Lower & Upper \\
\hline Support Effectiveness & Intercept SD & 0.34 & 0.18 & 0.02 & 0.65 \\
Support Effectiveness & RES SD & 0.31 & 0.11 & 0.08 & 0.51 \\
Support Effectiveness & PR SD & 0.29 & 0.12 & 0.05 & 0.52 \\
Support Effectiveness & Intercept-RES Cor & -0.17 & 0.40 & -0.85 & 0.71 \\
Support Effectiveness & Intercept-PR Cor & -0.03 & 0.43 & -0.81 & 0.81 \\
Support Effectiveness & RES-PR Cor & -0.52 & 0.35 & -0.95 & 0.40 \\
Support Effectiveness & Residual & 1.16 & 0.06 & 1.05 & 1.27 \\
Support Effectiveness & AR(1) & 0.16 & 0.09 & -0.03 & 0.33 \\
\hline Negative Mood & Intercept SD & 0.82 & 0.11 & 0.59 & 1.00 \\
Negative Mood & RES SD & 0.20 & 0.10 & 0.02 & 0.39 \\
Negative Mood & PR SD & 0.27 & 0.11 & 0.04 & 0.46 \\
Negative Mood & Intercept-RES Cor & -0.28 & 0.33 & -0.86 & 0.47 \\
Negative Mood & Intercept-PR Cor & -0.01 & 0.32 & -0.64 & 0.65 \\
Negative Mood & RES-PR Cor & -0.09 & 0.43 & -0.83 & 0.78 \\
Negative Mood & Residual & 1.04 & 0.05 & 0.94 & 1.15 \\
Negative Mood & AR(1) & 0.24 & 0.11 & 0.03 & 0.45 \\
\hline Positive Mood & Intercept SD & 0.55 & 0.18 & 0.09 & 0.81 \\
Positive Mood & RES SD & 0.14 & 0.09 & 0.007 & 0.35 \\
Positive Mood & PR SD & 0.16 & 0.11 & 0.008 & 0.39 \\
Positive Mood & Intercept-RES Cor & -0.19 & 0.44 & -0.91 & 0.75 \\
Positive Mood & Intercept-PR Cor & 0.17 & 0.44 & -0.75 & 0.89 \\
Positive Mood & RES-PR Cor & -0.18 & 0.50 & -0.93 & 0.81 \\
Positive Mood & Residual & 1.24 & 0.06 & 1.12 & 1.36 \\
Positive Mood & AR(1) & 0.15 & 0.11 & -0.06 & 0.36 \\
\hline Note & & & & \\
\hline
\end{tabular}

Note. $\mathrm{RES}=$ Regulatory Effectiveness of Support. $\mathrm{PR}=$ Perceived Responsiveness. IOS $=$ Inclusion of the other in the self. $\mathrm{SD}=$ standard deviation. Cor $=$ correlation . 


\section{Table S13B}

Summary of random effects, Study 5, continued

\begin{tabular}{llcccc}
\hline dv & term & Estimate & SE & Lower & Upper \\
\hline Coping & Intercept SD & 0.82 & 0.07 & 0.66 & 0.95 \\
Coping & RES SD & 0.08 & 0.06 & 0.003 & 0.20 \\
Coping & PR SD & 0.08 & 0.06 & 0.004 & 0.21 \\
Coping & Intercept-RES Cor & 0.19 & 0.42 & -0.71 & 0.87 \\
Coping & Intercept-PR Cor & -0.27 & 0.42 & -0.91 & 0.70 \\
Coping & RES-PR Cor & -0.16 & 0.50 & -0.92 & 0.83 \\
Coping & Residual & 0.80 & 0.04 & 0.73 & 0.88 \\
Coping & AR(1) & 0.14 & 0.11 & -0.06 & 0.38 \\
\hline IOS & Intercept SD & 1.07 & 0.10 & 0.87 & 1.26 \\
IOS & RES SD & 0.10 & 0.07 & 0.004 & 0.26 \\
IOS & PR SD & 0.38 & 0.07 & 0.23 & 0.53 \\
IOS & Intercept-RES Cor & -0.06 & 0.43 & -0.84 & 0.79 \\
IOS & Intercept-PR Cor & -0.03 & 0.19 & -0.40 & 0.36 \\
IOS & RES-PR Cor & -0.06 & 0.46 & -0.86 & 0.82 \\
IOS & Residual & 0.95 & 0.05 & 0.84 & 1.06 \\
IOS & AR(1) & 0.37 & 0.12 & 0.13 & 0.61 \\
\hline Sleep Quality & Intercept SD & 1.08 & 0.15 & 0.75 & 1.35 \\
Sleep Quality & RES SD & 0.16 & 0.10 & 0.007 & 0.38 \\
Sleep Quality & PR SD & 0.19 & 0.13 & 0.01 & 0.49 \\
Sleep Quality & Intercept-RES Cor & 0.11 & 0.41 & -0.73 & 0.86 \\
Sleep Quality & Intercept-PR Cor & -0.16 & 0.41 & -0.86 & 0.71 \\
Sleep Quality & RES-PR Cor & -0.29 & 0.49 & -0.95 & 0.78 \\
Sleep Quality & Residual & 1.00 & 0.10 & 0.82 & 1.22 \\
Sleep Quality & AR(1) & 0.17 & 0.19 & -0.20 & 0.54 \\
\hline
\end{tabular}

Note. RES = Regulatory Effectiveness of Support. PR = Perceived Responsiveness. IOS $=$ Inclusion of the other in the self. SD $=$ standard deviation. Cor $=$ correlation. 


\section{Table S13C}

Summary of random effects, Study 5, continued

\begin{tabular}{llcccc}
\hline $\mathrm{dv}$ & term & Estimate & SE & Lower & Upper \\
\hline Task Motivation & Intercept SD & 1.25 & 0.10 & 1.05 & 1.43 \\
Task Motivation & RES SD & 0.25 & 0.08 & 0.10 & 0.39 \\
Task Motivation & PR SD & 0.13 & 0.08 & 0.008 & 0.30 \\
Task Motivation & Intercept-RES Cor & -0.49 & 0.24 & -0.90 & -0.004 \\
Task Motivation & Intercept-PR Cor & -0.27 & 0.40 & -0.90 & 0.65 \\
Task Motivation & RES-PR Cor & 0.31 & 0.43 & -0.64 & 0.93 \\
Task Motivation & Residual & 0.99 & 0.06 & 0.89 & 1.10 \\
Task Motivation & AR(1) & 0.29 & 0.12 & 0.07 & 0.53 \\
\hline Task Performance & Intercept SD & 0.89 & 0.12 & 0.64 & 1.10 \\
Task Performance & RES SD & 0.12 & 0.08 & 0.005 & 0.29 \\
Task Performance & PR SD & 0.07 & 0.05 & 0.003 & 0.20 \\
Task Performance & Intercept-RES Cor & -0.12 & 0.40 & -0.86 & 0.72 \\
Task Performance & Intercept-PR Cor & -0.02 & 0.45 & -0.85 & 0.83 \\
Task Performance & RES-PR Cor & -0.19 & 0.50 & -0.93 & 0.82 \\
Task Performance & Residual & 0.91 & 0.06 & 0.79 & 1.03 \\
Task Performance & AR(1) & 0.33 & 0.15 & 0.04 & 0.61 \\
\hline
\end{tabular}

Note. RES = Regulatory Effectiveness of Support. $\mathrm{PR}=$ Perceived Responsiveness. IOS $=$ Inclusion of the other in the self. $\mathrm{SD}=$ standard deviation. Cor $=$ correlation. 


\section{Additional Speech Performance Variables, Study 7}

As discussed in Footnote 12, our speech performance coding scheme also included measures to assess verbal indicators of speech quality $(\mathrm{ICC}(\mathrm{A}, 3)=0.87)$, such as the fluidity and persuasiveness of the speech, and emotional expressions during the speech (higher numbers indicate more positivity and less negativity; $(\operatorname{ICC}(\mathrm{A}, 3)=0.71)$. Results for these variables are presented below.

\section{Table S14}

Summary of Results for Additional Speech Variables, Study 7

\begin{tabular}{llcccccc}
\hline DV & Predictor & Estimate & SE & Lower & Upper & Post_Prob & N_Subj \\
\hline Verbal Speech Quality & Intercept & 4.41 & 0.10 & 4.20 & 4.62 & - & 106 \\
Verbal Speech Quality & RES & -0.04 & 0.13 & -0.29 & 0.21 & 0.37 & 106 \\
Verbal Speech Quality & PR & -0.06 & 0.13 & -0.32 & 0.21 & 0.34 & 106 \\
\hline Emotional Expression & Intercept & 4.11 & 0.04 & 4.03 & 4.20 & - & 106 \\
Emotional Expression & RES & 0.02 & 0.05 & -0.09 & 0.12 & 0.63 & 106 \\
Emotional Expression & PR & 0.05 & 0.06 & -0.06 & 0.16 & 0.82 & 106 \\
\hline
\end{tabular}

Note. RES = Regulatory Effectiveness of Support. PR = Perceived Responsiveness. Lower and Upper refer to $95 \%$ credibility intervals. Post_Prob is the posterior probability that the effect is in the hypothesized direction.

\section{Table S15}

Differences in Effects of RES and PR for Additional Speech Variables, Study 7

\begin{tabular}{lccccl}
\hline DV & RES-PR & SE & Lower & Upper & Post_Prob \\
\hline Verbal Speech Quality & 0.01 & 0.23 & -0.45 & 0.48 & 0.52 \\
Emotional Expression & -0.03 & 0.10 & -0.23 & 0.16 & 0.37 \\
\hline
\end{tabular}

Note. RES = Regulatory Effectiveness of Support. PR = Perceived Responsiveness. Lower and Upper refer to $95 \%$ credibility intervals.

Post_Prob is the posterior probability that the effect is in the hypothesized direction. 


\section{Cardiovascular Results Tables, Study 7}

\section{Table S16}

Summary of results for cardiovascular data, Study 7

\begin{tabular}{lccccccc}
\hline Coefficient & Estimate & SE & Lower & Upper & Post_Prob & N_Subj & N_Obs \\
\hline Intercept & 670.07 & 11.18 & 647.89 & 691.74 & - & 106 & 3226 \\
RES & 11.18 & 12.45 & -13.57 & 35.26 & 0.82 & 106 & 3226 \\
PR & -18.64 & 13.45 & -44.53 & 8.49 & 0.08 & 106 & 3226 \\
Baseline vs. Speech & 135.50 & 10.54 & 115.32 & 156.54 & 1.00 & 106 & 3226 \\
Support vs. Speech & 58.22 & 6.37 & 45.77 & 70.63 & 1.00 & 106 & 3226 \\
Recovery vs. Speech & 137.24 & 8.54 & 120.46 & 154.12 & 1.00 & 106 & 3226 \\
RES x Baseline vs. Speech & -6.90 & 11.75 & -30.55 & 15.65 & 0.72 & 106 & 3226 \\
RES x Support vs. Speech & $\mathbf{- 1 1 . 4 4}$ & $\mathbf{7 . 2 3}$ & $\mathbf{- 2 5 . 4 7}$ & $\mathbf{2 . 6 2}$ & $\mathbf{0 . 9 4}$ & $\mathbf{1 0 6}$ & $\mathbf{3 2 2 6}$ \\
RES x Recovery vs. Speech & -9.08 & 9.72 & -28.49 & 10.14 & 0.83 & 106 & 3226 \\
PR x Baseline vs. Speech & 0.68 & 12.49 & -23.86 & 25.62 & 0.48 & 106 & 3226 \\
PR x Support vs. Speech & 8.03 & 7.60 & -7.14 & 22.88 & 0.14 & 106 & 3226 \\
PR x Recovery vs. Speech & 4.00 & 10.25 & -15.95 & 24.06 & 0.34 & 106 & 3226 \\
\hline
\end{tabular}

Note. RES = Regulatory Effectiveness of Support. PR = Perceived Responsiveness. For contrasts, Speech is coded as 0, otherwise 1. Lower and Upper refer to $95 \%$ credibility intervals. Post_Prob is the posterior probability that the effect is in the hypothesized direction. 


\section{Table S17}

Summary of random effects for cardiovascular data, Study 7

\begin{tabular}{lcccc}
\hline Term & Estimate & SE & Lower & Upper \\
\hline Intercept SD & 111.71 & 8.53 & 96.19 & 129.85 \\
Baseline vs. Speech SD & 100.55 & 7.68 & 86.61 & 116.54 \\
Support vs. Speech SD & 56.97 & 5.00 & 47.83 & 67.55 \\
Recovery vs. Speech SD & 79.63 & 6.76 & 67.43 & 93.63 \\
Intercept-Baseline vs. Speech Cor & -0.27 & 0.10 & -0.46 & -0.08 \\
Intercept-Support vs, Speech Cor & -0.20 & 0.11 & -0.41 & 0.01 \\
Intercept-Recovery vs. Speech Cor & -0.19 & 0.11 & -0.39 & 0.03 \\
Support vs. Speech-Baseline vs. Speech Cor & 0.68 & 0.07 & 0.53 & 0.79 \\
Support vs. Speech-Recovery vs. Speech Cor & 0.62 & 0.08 & 0.44 & 0.76 \\
Recovery vs. Speech-Baseline vs. Speech Cor & 0.81 & 0.04 & 0.71 & 0.88 \\
Residual & 39.66 & 0.56 & 38.58 & 40.78 \\
AR(1) & 0.39 & 0.03 & 0.34 & 0.45 \\
\hline
\end{tabular}

Note. $\mathrm{RES}=$ Regulatory Effectiveness of Support. $\mathrm{PR}=$ Perceived Responsiveness. For contrasts, Speech is coded as 0 , and the comparison phase is coded as 1 . 


\section{Meta-Analysis without Support Effectiveness}

We performed additional versions of our analyses without support effectiveness included in order to reduce potential issues of content overlap with RES and PR.

\section{Table S18}

Summary of supplemental meta-analysis results, fixed effects

\begin{tabular}{llccccc}
\hline Variable & Coefficient & Estimate & SE & Lower & Upper & N_Obs \\
\hline RES & Intercept & 0.23 & 0.05 & 0.14 & 0.35 & 46 \\
RES & Relational Variable & -0.05 & 0.09 & -0.26 & 0.10 & 46 \\
RES & Level of Analysis & -0.08 & 0.03 & -0.14 & -0.02 & 46 \\
\hline PR & Intercept & 0.05 & 0.04 & -0.04 & 0.14 & 46 \\
PR & Relational Variable & 0.40 & 0.06 & 0.28 & 0.52 & 46 \\
PR & Level of Analysis & -0.02 & 0.03 & -0.08 & 0.04 & 46 \\
\hline RES-PR & Intercept & 0.15 & 0.05 & 0.06 & 0.25 & 46 \\
RES-PR & Relational Variable & -0.42 & 0.07 & -0.56 & -0.29 & 46 \\
RES-PR & Level of Analysis & -0.06 & 0.04 & -0.15 & 0.02 & 46 \\
\hline
\end{tabular}

Note. RES = Regulatory Effectiveness of Support. PR = Perceived Responsiveness. RES-PR = difference of RES and PR (RES minus PR). Relational Variable is coded as $1=$ relational variable, $0=$ non-relational variable (self-regulation relevant variable). Level of Analysis is coded as $1=$ within-person effect, $0=$ between-person. 


\section{Table S19}

Summary of supplemental meta-analysis results without support effectiveness, random effects

\begin{tabular}{llcccc}
\hline Model & Term & Estimate & SE & Lower & Upper \\
\hline RES & DV SD & 0.07 & 0.06 & 0.00 & 0.23 \\
RES & Study SD & 0.05 & 0.05 & 0.00 & 0.17 \\
RES & DV x Study SD & 0.03 & 0.02 & 0.00 & 0.09 \\
RES & Residual & 0.04 & 0.02 & 0.00 & 0.09 \\
\hline PR & DV SD & 0.04 & 0.04 & 0.00 & 0.13 \\
PR & Study SD & 0.05 & 0.05 & 0.00 & 0.19 \\
PR & DV x Study SD & 0.02 & 0.01 & 0.00 & 0.06 \\
PR & Residual & 0.05 & 0.02 & 0.01 & 0.09 \\
\hline RES-PR & DV SD & 0.04 & 0.04 & 0.00 & 0.14 \\
RES-PR & Study SD & 0.04 & 0.04 & 0.00 & 0.14 \\
RES-PR & DV x Study SD & 0.03 & 0.02 & 0.00 & 0.08 \\
RES-PR & Residual & 0.05 & 0.03 & 0.00 & 0.12 \\
\hline
\end{tabular}

Note. $\mathrm{RES}=$ Regulatory Effectiveness of Support. PR = Perceived Responsiveness. RES-PR = difference of RES and PR (RES minus PR). Relational Variable is coded as $1=$ relational variable, $0=$ non-relational variable (self-regulation relevant variable). Level of Analysis is coded as $1=$ within-person effect, $0=$ between-person. SD $=$ standard deviation. 


\section{Results Summaries for Models with RES and PR Analyzed Separately}

The following tables show the results of the main analyses with the effects of RES and PR modeled separately. All results present unstandardized coefficients, which are posterior means. Lower and Upper refer to $95 \%$ credibility intervals. Post_Prob is the posterior probability that the effect is in the hypothesized direction.

\section{Table S20}

Effects of RES, Study 2

\begin{tabular}{llcccccc}
\hline DV & Predictor & Estimate & SE & Lower & Upper & Post_Prob & N_Subj \\
\hline Negative Mood & Intercept & 1.71 & 0.07 & 1.58 & 1.84 & - & 181 \\
Negative Mood & RES & -0.45 & 0.07 & -0.58 & -0.32 & 1.00 & 181 \\
\hline Positive Mood & Intercept & 5.03 & 0.08 & 4.87 & 5.20 & - & 181 \\
Positive Mood & RES & 0.79 & 0.08 & 0.62 & 0.95 & 1.00 & 181 \\
\hline IOS & Intercept & 5.99 & 0.07 & 5.85 & 6.13 & - & 181 \\
IOS & RES & 0.48 & 0.07 & 0.34 & 0.63 & 1.00 & 181 \\
\hline
\end{tabular}

\section{Table S21}

Effects of PR, Study 2

\begin{tabular}{llcccccc}
\hline DV & Predictor & Estimate & SE & Lower & Upper & Post_Prob & N_Subj \\
\hline Negative Mood & Intercept & 1.70 & 0.07 & 1.56 & 1.84 & - & 181 \\
Negative Mood & PR & -0.44 & 0.08 & -0.61 & -0.28 & 1.00 & 181 \\
\hline Positive Mood & Intercept & 5.04 & 0.09 & 4.87 & 5.20 & - & 181 \\
Positive Mood & PR & 0.85 & 0.11 & 0.65 & 1.06 & 1.00 & 181 \\
\hline IOS & Intercept & 6.00 & 0.07 & 5.87 & 6.13 & - & 181 \\
IOS & PR & 0.71 & 0.08 & 0.55 & 0.87 & 1.00 & 181 \\
\hline
\end{tabular}




\section{Table S22}

Fixed Effects of RES, Study 3

\begin{tabular}{llcccccc}
\hline DV & Predictor & Estimate & SE & Lower & Upper & N_Subj & N_Obs \\
\hline Support Effectiveness & Intercept & 4.29 & 0.06 & 4.17 & 4.41 & 222 & 720 \\
Support Effectiveness & RES-within & 0.57 & 0.05 & 0.47 & 0.67 & 222 & 720 \\
Support Effectiveness & RES-between & 0.75 & 0.05 & 0.65 & 0.86 & 222 & 720 \\
Support Effectiveness & Day & -0.05 & 0.03 & -0.12 & 0.01 & 222 & 720 \\
\hline Negative Mood & Intercept & 2.95 & 0.07 & 2.81 & 3.10 & 222 & 729 \\
Negative Mood & RES-within & -0.24 & 0.04 & -0.32 & -0.16 & 222 & 729 \\
Negative Mood & RES-between & -0.22 & 0.06 & -0.34 & -0.09 & 222 & 729 \\
Negative Mood & Day & -0.14 & 0.03 & -0.20 & -0.09 & 222 & 729 \\
\hline Positive Mood & Intercept & 3.66 & 0.07 & 3.53 & 3.79 & 222 & 729 \\
Positive Mood & RES-within & 0.24 & 0.04 & 0.16 & 0.32 & 222 & 729 \\
Positive Mood & RES-between & 0.25 & 0.06 & 0.13 & 0.37 & 222 & 729 \\
Positive Mood & Day & 0.01 & 0.03 & -0.05 & 0.08 & 222 & 729 \\
\hline Coping & Intercept & 4.42 & 0.05 & 4.31 & 4.53 & 222 & 729 \\
Coping & RES-within & 0.12 & 0.03 & 0.07 & 0.18 & 222 & 729 \\
Coping & RES-between & 0.13 & 0.05 & 0.04 & 0.22 & 222 & 729 \\
Coping & Day & -0.12 & 0.02 & -0.17 & -0.07 & 222 & 729 \\
\hline IOS & Intercept & 4.24 & 0.09 & 4.05 & 4.42 & 222 & 729 \\
IOS & RES-within & 0.32 & 0.04 & 0.23 & 0.40 & 222 & 729 \\
IOS & RES-between & 0.60 & 0.08 & 0.44 & 0.76 & 222 & 729 \\
IOS & Day & 0.003 & 0.03 & -0.05 & 0.06 & 222 & 729 \\
\hline
\end{tabular}




\section{Table S23}

Fixed Effects of PR, Study 3

\begin{tabular}{llccccccc}
\hline DV & Predictor & Estimate & SE & Lower & Upper & N_Subj & N_Obs \\
\hline Support Effectiveness & Intercept & 4.29 & 0.07 & 4.16 & 4.42 & 226 & 736 \\
Support Effectiveness & PR-within & 0.53 & 0.06 & 0.42 & 0.64 & 226 & 736 \\
Support Effectiveness & PR-between & 0.63 & 0.05 & 0.53 & 0.73 & 226 & 736 \\
Support Effectiveness & Day & -0.07 & 0.04 & -0.13 & 0.002 & 226 & 736 \\
\hline Negative Mood & Intercept & 2.92 & 0.07 & 2.78 & 3.06 & 227 & 758 \\
Negative Mood & PR-within & -0.16 & 0.05 & -0.26 & -0.07 & 227 & 758 \\
Negative Mood & PR-between & -0.18 & 0.06 & -0.28 & -0.07 & 227 & 758 \\
Negative Mood & Day & -0.13 & 0.03 & -0.19 & -0.08 & 227 & 758 \\
\hline Positive Mood & Intercept & 3.68 & 0.07 & 3.54 & 3.81 & 227 & 758 \\
Positive Mood & PR-within & 0.21 & 0.05 & 0.11 & 0.30 & 227 & 758 \\
Positive Mood & PR-between & 0.11 & 0.05 & 0.007 & 0.21 & 227 & 758 \\
Positive Mood & Day & 0.004 & 0.03 & -0.06 & 0.07 & 227 & 758 \\
\hline Coping & Intercept & 4.37 & 0.06 & 4.26 & 4.49 & 227 & 758 \\
Coping & PR-within & 0.08 & 0.03 & 0.02 & 0.15 & 227 & 758 \\
Coping & PR-between & 0.11 & 0.04 & 0.03 & 0.19 & 227 & 758 \\
Coping & Day & -0.13 & 0.02 & -0.18 & -0.08 & 227 & 758 \\
\hline IOS & Intercept & 4.25 & 0.08 & 4.08 & 4.41 & 227 & 758 \\
IOS & PR-within & 0.51 & 0.04 & 0.43 & 0.60 & 227 & 758 \\
IOS & PR-between & 0.68 & 0.06 & 0.55 & 0.80 & 227 & 758 \\
IOS & Day & 0.01 & 0.03 & -0.04 & 0.06 & 227 & 758 \\
\hline
\end{tabular}




\section{Table S24}

Fixed Effects of RES, Study 4

\begin{tabular}{llcccccc}
\hline DV & Predictor & Estimate & SE & Lower & Upper & N_Subj & N_Obs \\
\hline Support Effectiveness & Intercept & 4.30 & 0.08 & 4.15 & 4.45 & 176 & 538 \\
Support Effectiveness & RES-within & 0.65 & 0.06 & 0.53 & 0.77 & 176 & 538 \\
Support Effectiveness & RES-between & 0.71 & 0.06 & 0.60 & 0.81 & 176 & 538 \\
Support Effectiveness & Day & -0.01 & 0.04 & -0.08 & 0.06 & 176 & 538 \\
\hline Negative Mood & Intercept & 3.04 & 0.09 & 2.87 & 3.21 & 176 & 548 \\
Negative Mood & RES-within & -0.20 & 0.05 & -0.30 & -0.10 & 176 & 548 \\
Negative Mood & RES-between & -0.32 & 0.06 & -0.45 & -0.20 & 176 & 548 \\
Negative Mood & Day & -0.13 & 0.03 & -0.20 & -0.07 & 176 & 548 \\
\hline Positive Mood & Intercept & 3.79 & 0.07 & 3.65 & 3.93 & 176 & 548 \\
Positive Mood & RES-within & 0.15 & 0.05 & 0.05 & 0.26 & 176 & 548 \\
Positive Mood & RES-between & 0.37 & 0.06 & 0.26 & 0.49 & 176 & 548 \\
Positive Mood & Day & 0.03 & 0.03 & -0.03 & 0.10 & 176 & 548 \\
\hline Coping & Intercept & 4.38 & 0.07 & 4.25 & 4.52 & 176 & 549 \\
Coping & RES-within & 0.17 & 0.04 & 0.09 & 0.24 & 176 & 549 \\
Coping & RES-between & 0.16 & 0.05 & 0.06 & 0.26 & 176 & 549 \\
Coping & Day & -0.18 & 0.03 & -0.23 & -0.13 & 176 & 549 \\
\hline IOS & Intercept & 4.12 & 0.11 & 3.92 & 4.33 & 176 & 549 \\
IOS & RES-within & 0.32 & 0.05 & 0.22 & 0.43 & 176 & 549 \\
IOS & RES-between & 0.72 & 0.08 & 0.56 & 0.88 & 176 & 549 \\
IOS & Day & -0.05 & 0.03 & -0.11 & 0.01 & 176 & 549 \\
\hline Sleep Quality & Intercept & 4.98 & 0.11 & 4.76 & 5.20 & 131 & 288 \\
Sleep Quality & RES-within (lagged) & 0.07 & 0.08 & -0.09 & 0.23 & 131 & 288 \\
Sleep Quality & RES-between & 0.21 & 0.09 & 0.04 & 0.38 & 131 & 288 \\
Sleep Quality & Day & -0.02 & 0.06 & -0.15 & 0.10 & 131 & 288 \\
\hline
\end{tabular}




\section{Table S25}

Fixed Effects of PR, Study 4

\begin{tabular}{llcccccc}
\hline DV & Predictor & Estimate & SE & Lower & Upper & N_Subj & N_Obs \\
\hline Support Effectiveness & Intercept & 4.29 & 0.07 & 4.15 & 4.43 & 177 & 546 \\
Support Effectiveness & PR-within & 0.65 & 0.06 & 0.52 & 0.77 & 177 & 546 \\
Support Effectiveness & PR-between & 0.72 & 0.06 & 0.61 & 0.83 & 177 & 546 \\
Support Effectiveness & Day & 0.02 & 0.04 & -0.05 & 0.10 & 177 & 546 \\
\hline Negative Mood & Intercept & 3.02 & 0.09 & 2.85 & 3.19 & 179 & 574 \\
Negative Mood & PR-within & -0.19 & 0.06 & -0.30 & -0.07 & 179 & 574 \\
Negative Mood & PR-between & -0.25 & 0.07 & -0.39 & -0.11 & 179 & 574 \\
Negative Mood & Day & -0.14 & 0.03 & -0.21 & -0.08 & 179 & 574 \\
\hline Positive Mood & Intercept & 3.81 & 0.08 & 3.65 & 3.96 & 179 & 574 \\
Positive Mood & PR-within & 0.20 & 0.06 & 0.09 & 0.31 & 179 & 574 \\
Positive Mood & PR-between & 0.21 & 0.06 & 0.09 & 0.33 & 179 & 574 \\
Positive Mood & Day & 0.05 & 0.03 & -0.02 & 0.11 & 179 & 574 \\
\hline Coping & Intercept & 4.35 & 0.07 & 4.21 & 4.50 & 179 & 575 \\
Coping & PR-within & 0.14 & 0.04 & 0.06 & 0.23 & 179 & 575 \\
Coping & PR-between & 0.13 & 0.05 & 0.03 & 0.24 & 179 & 575 \\
Coping & Day & -0.16 & 0.03 & -0.21 & -0.10 & 179 & 575 \\
\hline IOS & Intercept & 4.10 & 0.10 & 3.91 & 4.30 & 179 & 575 \\
IOS & PR-within & 0.38 & 0.04 & 0.29 & 0.46 & 179 & 575 \\
IOS & PR-between & 0.85 & 0.07 & 0.70 & 0.99 & 179 & 575 \\
IOS & Day & -0.04 & 0.03 & -0.10 & 0.01 & 179 & 575 \\
\hline Sleep Quality & Intercept & 5.01 & 0.11 & 4.79 & 5.22 & 135 & 317 \\
Sleep Quality & PR-within (lagged) & -0.01 & 0.08 & -0.16 & 0.13 & 135 & 317 \\
Sleep Quality & PR-between & 0.16 & 0.09 & -0.02 & 0.34 & 135 & 317 \\
Sleep Quality & Day & -0.05 & 0.06 & -0.16 & 0.06 & 135 & 317 \\
\hline & & & & & & & \\
\hline
\end{tabular}




\section{Table S26}

Fixed Effects of RES, Study 5

\begin{tabular}{|c|c|c|c|c|c|c|c|}
\hline $\mathrm{DV}$ & Predictor & Estimate & $\mathrm{SE}$ & Lower & Upper & $\mathrm{N}_{\text {_Subj }}$ & $\mathrm{N} \_\mathrm{Obs}$ \\
\hline Support Effectiveness & Intercept & 4.54 & 0.07 & 4.40 & 4.67 & 231 & 624 \\
\hline Support Effectiveness & RES-within & 0.56 & 0.06 & 0.45 & 0.67 & 231 & 624 \\
\hline Support Effectiveness & RES-between & 0.63 & 0.05 & 0.53 & 0.74 & 231 & 624 \\
\hline Support Effectiveness & Day & 0.001 & 0.04 & -0.07 & 0.07 & 231 & 624 \\
\hline Negative Mood & Intercept & 2.93 & 0.08 & 2.78 & 3.08 & 231 & 636 \\
\hline Negative Mood & RES-within & -0.16 & 0.05 & -0.25 & -0.06 & 231 & 636 \\
\hline Negative Mood & RES-between & -0.13 & 0.06 & -0.24 & -0.03 & 231 & 636 \\
\hline Negative Mood & Day & -0.20 & 0.03 & -0.27 & -0.14 & 231 & 636 \\
\hline Positive Mood & Intercept & 3.80 & 0.07 & 3.67 & 3.93 & 232 & 639 \\
\hline Positive Mood & RES-within & 0.13 & 0.05 & 0.03 & 0.23 & 232 & 639 \\
\hline Positive Mood & RES-between & 0.29 & 0.05 & 0.19 & 0.39 & 232 & 639 \\
\hline Positive Mood & Day & 0.05 & 0.04 & -0.02 & 0.12 & 232 & 639 \\
\hline Coping & Intercept & 4.44 & 0.07 & 4.31 & 4.57 & 232 & 643 \\
\hline Coping & RES-within & 0.11 & 0.03 & 0.05 & 0.18 & 232 & 643 \\
\hline Coping & RES-between & 0.11 & 0.05 & 0.01 & 0.21 & 232 & 643 \\
\hline Coping & Day & -0.12 & 0.02 & -0.17 & -0.07 & 232 & 643 \\
\hline IOS & Intercept & 4.28 & 0.10 & 4.08 & 4.48 & 232 & 643 \\
\hline IOS & RES-within & 0.29 & 0.05 & 0.20 & 0.39 & 232 & 643 \\
\hline IOS & RES-between & 0.46 & 0.07 & 0.32 & 0.61 & 232 & 643 \\
\hline IOS & Day & 0.000 & 0.03 & -0.07 & 0.06 & 232 & 643 \\
\hline Sleep Quality & Intercept & 4.88 & 0.11 & 4.65 & 5.10 & 146 & 307 \\
\hline Sleep Quality & RES-within (lagged) & 0.04 & 0.07 & -0.09 & 0.17 & 146 & 307 \\
\hline Sleep Quality & RES-between & 0.19 & 0.09 & 0.006 & 0.38 & 146 & 307 \\
\hline Sleep Quality & Day & -0.02 & 0.06 & -0.13 & 0.09 & 146 & 307 \\
\hline Sleep Quality & sleeptime.wc & 0.30 & 0.05 & 0.20 & 0.41 & 146 & 307 \\
\hline Task Motivation & Intercept & 5.47 & 0.10 & 5.28 & 5.66 & 232 & 605 \\
\hline Task Motivation & RES-within & 0.11 & 0.05 & 0.01 & 0.21 & 232 & 605 \\
\hline Task Motivation & RES-between & 0.21 & 0.07 & 0.07 & 0.35 & 232 & 605 \\
\hline Task Motivation & Day & -0.05 & 0.03 & -0.12 & 0.01 & 232 & 605 \\
\hline Task Performance & Intercept & 5.06 & 0.08 & 4.90 & 5.21 & 232 & 605 \\
\hline Task Performance & RES-within & 0.03 & 0.04 & -0.05 & 0.11 & 232 & 605 \\
\hline Task Performance & RES-between & 0.22 & 0.06 & 0.11 & 0.33 & 232 & 605 \\
\hline Task Performance & Day & -0.06 & 0.03 & -0.12 & 0.001 & 232 & 605 \\
\hline
\end{tabular}




\section{Table S27}

Fixed Effects of PR, Study 5

\begin{tabular}{|c|c|c|c|c|c|c|c|}
\hline DV & Predictor & Estimate & SE & Lower & Upper & N_Subj & $\mathrm{N} \_$Obs \\
\hline Support Effectiveness & Intercept & 4.54 & 0.07 & 4.41 & 4.67 & 228 & 624 \\
\hline Support Effectiveness & PR-within & -0.13 & 0.09 & -0.31 & 0.04 & 228 & 624 \\
\hline Support Effectiveness & Day & 0.03 & 0.04 & -0.05 & 0.11 & 228 & 624 \\
\hline Support Effectiveness & ppr.c & 0.70 & 0.06 & 0.59 & 0.80 & 228 & 624 \\
\hline Negative Mood & Intercept & 2.93 & 0.07 & 2.78 & 3.07 & 228 & 651 \\
\hline Negative Mood & PR-within & -0.12 & 0.06 & -0.23 & -0.004 & 228 & 651 \\
\hline Negative Mood & PR-between & -0.15 & 0.06 & -0.27 & -0.03 & 228 & 651 \\
\hline Negative Mood & Day & -0.22 & 0.03 & -0.28 & -0.16 & 228 & 651 \\
\hline Positive Mood & Intercept & 3.78 & 0.07 & 3.64 & 3.93 & 229 & 655 \\
\hline Positive Mood & PR-within & 0.12 & 0.06 & 0.003 & 0.24 & 229 & 655 \\
\hline Positive Mood & PR-between & 0.22 & 0.06 & 0.11 & 0.34 & 229 & 655 \\
\hline Positive Mood & Day & 0.05 & 0.04 & -0.02 & 0.12 & 229 & 655 \\
\hline Coping & Intercept & 4.42 & 0.07 & 4.30 & 4.56 & 229 & 659 \\
\hline Coping & PR-within & 0.14 & 0.04 & 0.06 & 0.21 & 229 & 659 \\
\hline Coping & PR-between & 0.07 & 0.06 & -0.04 & 0.18 & 229 & 659 \\
\hline Coping & Day & -0.12 & 0.03 & -0.17 & -0.07 & 229 & 659 \\
\hline IOS & Intercept & 4.29 & 0.09 & 4.11 & 4.46 & 229 & 659 \\
\hline IOS & PR-within & 0.42 & 0.05 & 0.32 & 0.53 & 229 & 659 \\
\hline IOS & PR-between & 0.78 & 0.07 & 0.64 & 0.92 & 229 & 659 \\
\hline IOS & Day & 0.01 & 0.03 & -0.05 & 0.07 & 229 & 659 \\
\hline Sleep Quality & Intercept & 4.92 & 0.11 & 4.70 & 5.14 & 148 & 326 \\
\hline Sleep Quality & PR-within (lagged) & 0.07 & 0.07 & -0.08 & 0.21 & 148 & 326 \\
\hline Sleep Quality & PR-between & 0.19 & 0.10 & -0.01 & 0.38 & 148 & 326 \\
\hline Sleep Quality & Day & -0.04 & 0.05 & -0.15 & 0.06 & 148 & 326 \\
\hline Sleep Quality & sleeptime.wc & 0.38 & 0.05 & 0.28 & 0.47 & 148 & 326 \\
\hline Task Motivation & Intercept & 5.46 & 0.10 & 5.27 & 5.66 & 228 & 620 \\
\hline Task Motivation & PR-within & 0.08 & 0.05 & -0.02 & 0.19 & 228 & 620 \\
\hline Task Motivation & PR-between & 0.10 & 0.08 & -0.05 & 0.26 & 228 & 620 \\
\hline Task Motivation & Day & -0.02 & 0.03 & -0.09 & 0.04 & 228 & 620 \\
\hline Task Performance & Intercept & 5.05 & 0.08 & 4.89 & 5.20 & 228 & 620 \\
\hline Task Performance & PR-within & 0.007 & 0.04 & -0.07 & 0.09 & 228 & 620 \\
\hline Task Performance & PR-between & 0.13 & 0.07 & 0.008 & 0.26 & 228 & 620 \\
\hline Task Performance & Day & -0.05 & 0.03 & -0.10 & 0.004 & 228 & 620 \\
\hline
\end{tabular}




\section{Table S28}

Random Effects of RES, Study 3

\begin{tabular}{llcccc}
\hline DV & Term & Estimate & SE & Lower & Upper \\
\hline Support Effectiveness & Intercept SD & 0.39 & 0.17 & 0.03 & 0.67 \\
Support Effectiveness & RES SD & 0.28 & 0.08 & 0.09 & 0.42 \\
Support Effectiveness & Intercept-RES Cor & -0.22 & 0.40 & -0.91 & 0.69 \\
Support Effectiveness & Residual & 1.21 & 0.05 & 1.11 & 1.30 \\
Support Effectiveness & AR(1) & 0.24 & 0.08 & 0.08 & 0.39 \\
\hline Negative Mood & Intercept SD & 0.90 & 0.07 & 0.75 & 1.04 \\
Negative Mood & RES SD & 0.16 & 0.07 & 0.03 & 0.29 \\
Negative Mood & Intercept-RES Cor & -0.53 & 0.28 & -0.97 & 0.08 \\
Negative Mood & Residual & 1.04 & 0.04 & 0.96 & 1.12 \\
Negative Mood & AR(1) & 0.13 & 0.07 & -0.005 & 0.28 \\
\hline Positive Mood & Intercept SD & 0.72 & 0.12 & 0.45 & 0.91 \\
Positive Mood & RES SD & 0.09 & 0.07 & 0.004 & 0.25 \\
Positive Mood & Intercept-RES Cor & -0.22 & 0.47 & -0.95 & 0.82 \\
Positive Mood & Residual & 1.17 & 0.05 & 1.08 & 1.28 \\
Positive Mood & AR(1) & 0.10 & 0.09 & -0.06 & 0.30 \\
\hline Coping & Intercept SD & 0.52 & 0.14 & 0.16 & 0.72 \\
Coping & RES SD & 0.11 & 0.06 & 0.006 & 0.23 \\
Coping & Intercept-RES Cor & -0.04 & 0.41 & -0.86 & 0.83 \\
Coping & Residual & 0.83 & 0.05 & 0.75 & 0.92 \\
Coping & AR(1) & 0.32 & 0.14 & 0.07 & 0.57 \\
\hline IOS & Intercept SD & 1.26 & 0.08 & 1.10 & 1.42 \\
IOS & RES SD & 0.26 & 0.07 & 0.10 & 0.38 \\
IOS & Intercept-RES Cor & -0.12 & 0.20 & -0.50 & 0.26 \\
IOS & Residual & 0.99 & 0.04 & 0.91 & 1.08 \\
IOS & AR(1) & 0.11 & 0.08 & -0.04 & 0.27 \\
\hline & & & & & \\
\hline
\end{tabular}




\section{Table S29}

Random Effects of PR, Study 3

\begin{tabular}{llcccc}
\hline DV & Term & Estimate & SE & Lower & Upper \\
\hline Support Effectiveness & Intercept SD & 0.59 & 0.13 & 0.26 & 0.79 \\
Support Effectiveness & PR SD & 0.20 & 0.10 & 0.01 & 0.40 \\
Support Effectiveness & Intercept-PR Cor & -0.28 & 0.41 & -0.93 & 0.72 \\
Support Effectiveness & Residual & 1.26 & 0.05 & 1.17 & 1.37 \\
Support Effectiveness & AR(1) & 0.12 & 0.08 & -0.03 & 0.29 \\
\hline Negative Mood & Intercept SD & 0.84 & 0.08 & 0.68 & 1.00 \\
Negative Mood & PR SD & 0.22 & 0.09 & 0.02 & 0.39 \\
Negative Mood & Intercept-PR Cor & 0.06 & 0.32 & -0.61 & 0.72 \\
Negative Mood & Residual & 1.08 & 0.04 & 1.00 & 1.17 \\
Negative Mood & AR(1) & 0.21 & 0.07 & 0.07 & 0.35 \\
\hline Positive Mood & Intercept SD & 0.61 & 0.15 & 0.20 & 0.84 \\
Positive Mood & PR SD & 0.18 & 0.10 & 0.01 & 0.38 \\
Positive Mood & Intercept-PR Cor & 0.10 & 0.43 & -0.80 & 0.91 \\
Positive Mood & Residual & 1.20 & 0.05 & 1.10 & 1.31 \\
Positive Mood & AR(1) & 0.24 & 0.09 & 0.07 & 0.41 \\
\hline Coping & Intercept SD & 0.54 & 0.14 & 0.14 & 0.74 \\
Coping & PR SD & 0.06 & 0.04 & 0.002 & 0.16 \\
Coping & Intercept-PR Cor & -0.03 & 0.52 & -0.92 & 0.92 \\
Coping & Residual & 0.90 & 0.04 & 0.82 & 0.99 \\
Coping & AR(1) & 0.33 & 0.11 & 0.12 & 0.55 \\
\hline IOS & Intercept SD & 1.14 & 0.07 & 1.01 & 1.28 \\
IOS & PR SD & 0.18 & 0.08 & 0.02 & 0.31 \\
IOS & Intercept-PR Cor & -0.08 & 0.30 & -0.68 & 0.56 \\
IOS & Residual & 0.93 & 0.04 & 0.86 & 1.01 \\
IOS & AR(1) & 0.13 & 0.08 & -0.02 & 0.28 \\
\hline
\end{tabular}




\section{Table S30}

Random Effects of RES, Study 4

\begin{tabular}{|c|c|c|c|c|c|}
\hline DV & Term & Estimate & $\mathrm{SE}$ & Lower & Upper \\
\hline Support Effectiveness & Intercept SD & 0.71 & 0.09 & 0.52 & 0.88 \\
\hline Support Effectiveness & RES SD & 0.29 & 0.09 & 0.12 & 0.47 \\
\hline Support Effectiveness & Intercept-RES Cor & -0.68 & 0.21 & -0.98 & -0.22 \\
\hline Support Effectiveness & Residual & 1.10 & 0.05 & 1.00 & 1.21 \\
\hline Support Effectiveness & $\mathrm{AR}(1)$ & -0.02 & 0.09 & -0.19 & 0.18 \\
\hline Negative Mood & Intercept SD & 0.97 & 0.08 & 0.83 & 1.13 \\
\hline Negative Mood & RES SD & 0.20 & 0.07 & 0.08 & 0.34 \\
\hline Negative Mood & Intercept-RES Cor & -0.73 & 0.20 & -0.99 & -0.27 \\
\hline Negative Mood & Residual & 1.02 & 0.04 & 0.94 & 1.11 \\
\hline Negative Mood & $\mathrm{AR}(1)$ & -0.10 & 0.08 & -0.24 & 0.06 \\
\hline Positive Mood & Intercept SD & 0.77 & 0.07 & 0.64 & 0.91 \\
\hline Positive Mood & RES SD & 0.08 & 0.06 & 0.004 & 0.23 \\
\hline Positive Mood & Intercept-RES Cor & -0.02 & 0.52 & -0.91 & 0.92 \\
\hline Positive Mood & Residual & 1.10 & 0.05 & 1.02 & 1.20 \\
\hline Positive Mood & $\mathrm{AR}(1)$ & -0.26 & 0.08 & -0.40 & -0.10 \\
\hline Coping & Intercept SD & 0.75 & 0.06 & 0.62 & 0.88 \\
\hline Coping & RES SD & 0.15 & 0.08 & 0.02 & 0.30 \\
\hline Coping & Intercept-RES Cor & -0.34 & 0.34 & -0.94 & 0.38 \\
\hline Coping & Residual & 0.75 & 0.04 & 0.69 & 0.83 \\
\hline Coping & $\operatorname{AR}(1)$ & 0.04 & 0.09 & -0.12 & 0.24 \\
\hline IOS & Intercept SD & 1.25 & 0.09 & 1.09 & 1.44 \\
\hline IOS & RES SD & 0.34 & 0.07 & 0.22 & 0.47 \\
\hline IOS & Intercept-RES Cor & 0.03 & 0.17 & -0.30 & 0.35 \\
\hline IOS & Residual & 0.86 & 0.04 & 0.78 & 0.96 \\
\hline IOS & $\mathrm{AR}(1)$ & 0.10 & 0.11 & -0.09 & 0.33 \\
\hline Sleep Quality & Intercept SD & 0.98 & 0.11 & 0.77 & 1.18 \\
\hline Sleep Quality & RES SD & 0.17 & 0.10 & 0.007 & 0.38 \\
\hline Sleep Quality & Intercept-RES Cor & 0.37 & 0.43 & -0.71 & 0.96 \\
\hline Sleep Quality & Residual & 1.06 & 0.08 & 0.91 & 1.24 \\
\hline Sleep Quality & $\mathrm{AR}(1)$ & -0.28 & 0.13 & -0.51 & 0.01 \\
\hline
\end{tabular}




\section{Table S31}

Random Effects of PR, Study 4

\begin{tabular}{|c|c|c|c|c|c|}
\hline DV & Term & Estimate & $\mathrm{SE}$ & Lower & Upper \\
\hline Support Effectiveness & Intercept SD & 0.60 & 0.15 & 0.20 & 0.83 \\
\hline Support Effectiveness & PR SD & 0.15 & 0.11 & 0.006 & 0.39 \\
\hline Support Effectiveness & Intercept-PR Cor & -0.07 & 0.50 & -0.93 & 0.91 \\
\hline Support Effectiveness & Residual & 1.20 & 0.06 & 1.09 & 1.32 \\
\hline Support Effectiveness & $\mathrm{AR}(1)$ & 0.03 & 0.10 & -0.15 & 0.24 \\
\hline Negative Mood & Intercept SD & 1.00 & 0.08 & 0.85 & 1.17 \\
\hline Negative Mood & PR SD & 0.27 & 0.08 & 0.09 & 0.43 \\
\hline Negative Mood & Intercept-PR Cor & -0.27 & 0.23 & -0.73 & 0.20 \\
\hline Negative Mood & Residual & 1.02 & 0.04 & 0.93 & 1.11 \\
\hline Negative Mood & $\mathrm{AR}(1)$ & -0.001 & 0.08 & -0.15 & 0.17 \\
\hline Positive Mood & Intercept SD & 0.87 & 0.07 & 0.74 & 1.01 \\
\hline Positive Mood & PR SD & 0.11 & 0.08 & 0.005 & 0.28 \\
\hline Positive Mood & Intercept-PR Cor & 0.11 & 0.46 & -0.82 & 0.92 \\
\hline Positive Mood & Residual & 1.08 & 0.04 & 1.00 & 1.17 \\
\hline Positive Mood & $\mathrm{AR}(1)$ & -0.27 & 0.07 & -0.41 & -0.14 \\
\hline Coping & Intercept SD & 0.74 & 0.07 & 0.59 & 0.89 \\
\hline Coping & PR SD & 0.23 & 0.06 & 0.10 & 0.35 \\
\hline Coping & Intercept-PR Cor & -0.38 & 0.26 & -0.83 & 0.18 \\
\hline Coping & Residual & 0.83 & 0.04 & 0.76 & 0.91 \\
\hline Coping & $\mathrm{AR}(1)$ & 0.21 & 0.10 & 0.02 & 0.39 \\
\hline IOS & Intercept SD & 1.13 & 0.08 & 0.98 & 1.29 \\
\hline IOS & PR SD & 0.13 & 0.08 & 0.006 & 0.29 \\
\hline IOS & Intercept-PR Cor & -0.14 & 0.39 & -0.87 & 0.69 \\
\hline IOS & Residual & 0.89 & 0.04 & 0.81 & 0.97 \\
\hline IOS & $\mathrm{AR}(1)$ & 0.06 & 0.08 & -0.09 & 0.24 \\
\hline Sleep Quality & Intercept SD & 1.09 & 0.10 & 0.90 & 1.29 \\
\hline Sleep Quality & PR SD & 0.18 & 0.10 & 0.01 & 0.41 \\
\hline Sleep Quality & Intercept-PR Cor & 0.57 & 0.35 & -0.40 & 0.98 \\
\hline Sleep Quality & Residual & 0.99 & 0.06 & 0.88 & 1.13 \\
\hline Sleep Quality & $\mathrm{AR}(1)$ & -0.28 & 0.11 & -0.49 & -0.06 \\
\hline
\end{tabular}




\section{Table S32A}

Random Effects of RES, Study 5

\begin{tabular}{|c|c|c|c|c|c|}
\hline $\mathrm{dv}$ & term & Estimate & $\mathrm{SE}$ & Lower & Upper \\
\hline Support Effectiveness & Intercept SD & 0.54 & 0.17 & 0.10 & 0.80 \\
\hline Support Effectiveness & RES SD & 0.16 & 0.10 & 0.007 & 0.38 \\
\hline Support Effectiveness & Intercept-RES Cor & -0.13 & 0.49 & -0.94 & 0.89 \\
\hline Support Effectiveness & Residual & 1.25 & 0.06 & 1.13 & 1.37 \\
\hline Support Effectiveness & $\operatorname{AR}(1)$ & 0.16 & 0.09 & -0.02 & 0.34 \\
\hline Negative Mood & Intercept SD & 0.84 & 0.10 & 0.63 & 1.02 \\
\hline Negative Mood & RES SD & 0.24 & 0.09 & 0.05 & 0.39 \\
\hline Negative Mood & Intercept-RES Cor & -0.31 & 0.29 & -0.87 & 0.28 \\
\hline Negative Mood & Residual & 1.05 & 0.05 & 0.95 & 1.16 \\
\hline Negative Mood & $\operatorname{AR}(1)$ & 0.21 & 0.10 & 0.02 & 0.41 \\
\hline Positive Mood & Intercept SD & 0.52 & 0.18 & 0.06 & 0.79 \\
\hline Positive Mood & RES SD & 0.12 & 0.09 & 0.004 & 0.31 \\
\hline Positive Mood & Intercept-RES Cor & -0.09 & 0.51 & -0.94 & 0.90 \\
\hline Positive Mood & Residual & 1.26 & 0.06 & 1.15 & 1.38 \\
\hline Positive Mood & $\operatorname{AR}(1)$ & 0.17 & 0.10 & -0.03 & 0.36 \\
\hline Coping & Intercept SD & 0.83 & 0.07 & 0.70 & 0.96 \\
\hline Coping & RES SD & 0.07 & 0.05 & 0.003 & 0.19 \\
\hline Coping & Intercept-RES Cor & 0.17 & 0.48 & -0.86 & 0.94 \\
\hline Coping & Residual & 0.80 & 0.04 & 0.74 & 0.88 \\
\hline Coping & $\operatorname{AR}(1)$ & 0.12 & 0.10 & -0.06 & 0.33 \\
\hline IOS & Intercept SD & 1.25 & 0.10 & 1.04 & 1.44 \\
\hline IOS & RES SD & 0.22 & 0.10 & 0.02 & 0.39 \\
\hline IOS & Intercept-RES Cor & -0.25 & 0.30 & -0.85 & 0.37 \\
\hline IOS & Residual & 1.08 & 0.06 & 0.96 & 1.20 \\
\hline IOS & $\operatorname{AR}(1)$ & 0.31 & 0.10 & 0.11 & 0.51 \\
\hline Sleep Quality & Intercept SD & 1.05 & 0.15 & 0.74 & 1.31 \\
\hline Sleep Quality & RES SD & 0.11 & 0.08 & 0.005 & 0.30 \\
\hline Sleep Quality & Intercept-RES Cor & 0.22 & 0.50 & -0.85 & 0.96 \\
\hline Sleep Quality & Residual & 1.04 & 0.09 & 0.88 & 1.22 \\
\hline Sleep Quality & $\operatorname{AR}(1)$ & 0.21 & 0.16 & -0.10 & 0.53 \\
\hline
\end{tabular}




\section{Table S32B}

Random Effects of RES, Study 5, continued

\begin{tabular}{llcccc}
\hline $\mathrm{dv}$ & term & Estimate & SE & Lower & Upper \\
\hline Task Motivation & Intercept SD & 1.24 & 0.10 & 1.05 & 1.42 \\
Task Motivation & RES SD & 0.27 & 0.07 & 0.13 & 0.40 \\
Task Motivation & Intercept-RES Cor & -0.59 & 0.21 & -0.95 & -0.15 \\
Task Motivation & Residual & 0.99 & 0.05 & 0.89 & 1.10 \\
Task Motivation & AR(1) & 0.27 & 0.11 & 0.06 & 0.49 \\
\hline Task Performance & Intercept SD & 0.89 & 0.12 & 0.63 & 1.09 \\
Task Performance & RES SD & 0.09 & 0.06 & 0.004 & 0.24 \\
Task Performance & Intercept-RES Cor & -0.14 & 0.47 & -0.93 & 0.84 \\
Task Performance & Residual & 0.92 & 0.06 & 0.80 & 1.04 \\
Task Performance & AR(1) & 0.29 & 0.15 & 0.002 & 0.58 \\
\hline
\end{tabular}




\section{Table S33A}

Random Effects of PR, Study 5

\begin{tabular}{|c|c|c|c|c|c|}
\hline dv & term & Estimate & $\mathrm{SE}$ & Lower & Upper \\
\hline Support Effectiveness & Intercept SD & 0.37 & 0.18 & 0.03 & 0.67 \\
\hline Support Effectiveness & PR SD & 0.26 & 0.14 & 0.02 & 0.53 \\
\hline Support Effectiveness & Intercept-PR Cor & -0.07 & 0.47 & -0.92 & 0.86 \\
\hline Support Effectiveness & Residual & 1.30 & 0.06 & 1.18 & 1.41 \\
\hline Support Effectiveness & $\mathrm{AR}(1)$ & 0.13 & 0.08 & -0.04 & 0.28 \\
\hline Negative Mood & Intercept SD & 0.82 & 0.09 & 0.62 & 0.98 \\
\hline Negative Mood & PR SD & 0.31 & 0.08 & 0.14 & 0.47 \\
\hline Negative Mood & Intercept-PR Cor & -0.06 & 0.29 & -0.60 & 0.53 \\
\hline Negative Mood & Residual & 1.04 & 0.05 & 0.95 & 1.14 \\
\hline Negative Mood & $\mathrm{AR}(1)$ & 0.20 & 0.09 & 0.02 & 0.38 \\
\hline Positive Mood & Intercept SD & 0.57 & 0.17 & 0.12 & 0.81 \\
\hline Positive Mood & PR SD & 0.18 & 0.10 & 0.01 & 0.38 \\
\hline Positive Mood & Intercept-PR Cor & 0.24 & 0.44 & -0.76 & 0.95 \\
\hline Positive Mood & Residual & 1.25 & 0.06 & 1.15 & 1.37 \\
\hline Positive Mood & $\mathrm{AR}(1)$ & 0.17 & 0.10 & -0.02 & 0.37 \\
\hline Coping & Intercept SD & 0.80 & 0.08 & 0.62 & 0.95 \\
\hline Coping & PR SD & 0.06 & 0.04 & 0.002 & 0.16 \\
\hline Coping & Intercept-PR Cor & -0.18 & 0.51 & -0.96 & 0.88 \\
\hline Coping & Residual & 0.87 & 0.04 & 0.79 & 0.96 \\
\hline Coping & $\mathrm{AR}(1)$ & 0.21 & 0.10 & 0.04 & 0.43 \\
\hline IOS & Intercept SD & 1.03 & 0.10 & 0.82 & 1.22 \\
\hline IOS & PR SD & 0.31 & 0.07 & 0.16 & 0.45 \\
\hline IOS & Intercept-PR Cor & -0.10 & 0.23 & -0.53 & 0.38 \\
\hline IOS & Residual & 1.01 & 0.05 & 0.91 & 1.11 \\
\hline IOS & $\mathrm{AR}(1)$ & 0.41 & 0.10 & 0.21 & 0.60 \\
\hline Sleep Quality & Intercept SD & 1.12 & 0.12 & 0.86 & 1.35 \\
\hline Sleep Quality & PR SD & 0.11 & 0.08 & 0.005 & 0.32 \\
\hline Sleep Quality & Intercept-PR Cor & -0.06 & 0.52 & -0.93 & 0.92 \\
\hline Sleep Quality & Residual & 1.01 & 0.08 & 0.88 & 1.19 \\
\hline Sleep Quality & $\mathrm{AR}(1)$ & -0.05 & 0.16 & -0.34 & 0.29 \\
\hline
\end{tabular}


Table S33B

Random Effects of PR, Study 5, continued

\begin{tabular}{llcccc}
\hline $\mathrm{dv}$ & term & Estimate & SE & Lower & Upper \\
\hline Task Motivation & Intercept SD & 1.28 & 0.09 & 1.11 & 1.46 \\
Task Motivation & PR SD & 0.19 & 0.09 & 0.02 & 0.35 \\
Task Motivation & Intercept-PR Cor & -0.41 & 0.33 & -0.94 & 0.35 \\
Task Motivation & Residual & 1.02 & 0.05 & 0.92 & 1.12 \\
Task Motivation & AR(1) & 0.19 & 0.09 & 0.01 & 0.37 \\
\hline Task Performance & Intercept SD & 0.95 & 0.10 & 0.73 & 1.13 \\
Task Performance & PR SD & 0.06 & 0.05 & 0.002 & 0.18 \\
Task Performance & Intercept-PR Cor & 0.09 & 0.50 & -0.90 & 0.94 \\
Task Performance & Residual & 0.89 & 0.05 & 0.79 & 1.00 \\
Task Performance & AR(1) & 0.29 & 0.14 & 0.04 & 0.56 \\
\hline
\end{tabular}


Table S34

Effects of Self-Reported RES, Study 6

\begin{tabular}{llccccc}
\hline DV & Predictor & Estimate & SE & Lower & Upper & N_Subj \\
\hline Sup. Eff. & Intercept & 5.21 & 0.07 & 5.07 & 5.34 & 101 \\
Sup. Eff. & RES & 0.79 & 0.05 & 0.69 & 0.89 & 101 \\
\hline Negative Mood & Intercept & 2.63 & 0.10 & 2.44 & 2.84 & 101 \\
Negative Mood & RES & -0.30 & 0.07 & -0.45 & -0.15 & 101 \\
Negative Mood & Pre Neg. Mood & 0.47 & 0.06 & 0.36 & 0.58 & 101 \\
\hline Positive Mood & Intercept & 3.88 & 0.08 & 3.73 & 4.04 & 101 \\
Positive Mood & RES & 0.37 & 0.06 & 0.26 & 0.48 & 101 \\
Positive Mood & Pre Pos. Mood & 0.56 & 0.06 & 0.44 & 0.68 & 101 \\
\hline IOS & Intercept & 4.96 & 0.11 & 4.75 & 5.18 & 101 \\
IOS & RES & 0.52 & 0.08 & 0.37 & 0.68 & 101 \\
\hline Closeness & Intercept & 6.41 & 0.07 & 6.28 & 6.54 & 101 \\
Closeness & RES & 0.25 & 0.04 & 0.16 & 0.33 & 101 \\
Closeness & Pre Closeness & 0.35 & 0.08 & 0.19 & 0.51 & 101 \\
\hline
\end{tabular}


Table S35

Effects of Self-Reported PR, Study 6

\begin{tabular}{llccccc}
\hline DV & Predictor & Estimate & SE & Lower & Upper & N_Subj \\
\hline Sup. Eff. & Intercept & 5.21 & 0.10 & 5.02 & 5.39 & 101 \\
Sup. Eff. & PR & 0.65 & 0.08 & 0.51 & 0.80 & 101 \\
\hline Negative Mood & Intercept & 2.63 & 0.11 & 2.42 & 2.84 & 101 \\
Negative Mood & PR & -0.18 & 0.09 & -0.36 & 0.001 & 101 \\
Negative Mood & Pre Neg. Mood & 0.48 & 0.06 & 0.37 & 0.59 & 101 \\
\hline Positive Mood & Intercept & 3.89 & 0.08 & 3.73 & 4.05 & 101 \\
Positive Mood & PR & 0.30 & 0.07 & 0.16 & 0.45 & 101 \\
Positive Mood & Pre Pos. Mood & 0.61 & 0.06 & 0.48 & 0.73 & 101 \\
\hline IOS & Intercept & 4.96 & 0.10 & 4.76 & 5.16 & 101 \\
IOS & PR & 0.67 & 0.09 & 0.50 & 0.83 & 101 \\
\hline Closeness & Intercept & 6.41 & 0.06 & 6.29 & 6.52 & 101 \\
Closeness & PR & 0.43 & 0.05 & 0.33 & 0.52 & 101 \\
Closeness & Pre Closeness & 0.28 & 0.07 & 0.14 & 0.42 & 101 \\
\hline
\end{tabular}


Table S36

Effects of Coder-Rated RES, Study 6

\begin{tabular}{llccccc}
\hline DV & Predictor & Estimate & SE & Lower & Upper & N_Subj \\
\hline Sup. Eff. & Intercept & 5.21 & 0.07 & 5.07 & 5.34 & 101 \\
Sup. Eff. & RES & 0.79 & 0.05 & 0.69 & 0.89 & 101 \\
\hline Negative Mood & Intercept & 2.63 & 0.10 & 2.44 & 2.84 & 101 \\
Negative Mood & RES & -0.30 & 0.07 & -0.45 & -0.15 & 101 \\
Negative Mood & Pre Neg. Mood & 0.47 & 0.06 & 0.36 & 0.58 & 101 \\
\hline Positive Mood & Intercept & 3.88 & 0.08 & 3.73 & 4.04 & 101 \\
Positive Mood & RES & 0.37 & 0.06 & 0.26 & 0.48 & 101 \\
Positive Mood & Pre Pos. Mood & 0.56 & 0.06 & 0.44 & 0.68 & 101 \\
\hline IOS & Intercept & 4.96 & 0.11 & 4.75 & 5.18 & 101 \\
IOS & RES & 0.52 & 0.08 & 0.37 & 0.68 & 101 \\
\hline Closeness & Intercept & 6.41 & 0.07 & 6.28 & 6.54 & 101 \\
Closeness & RES & 0.25 & 0.04 & 0.16 & 0.33 & 101 \\
Closeness & Pre Closeness & 0.35 & 0.08 & 0.19 & 0.51 & 101 \\
\hline
\end{tabular}


Table S37

Effects of Coder-Rated PR, Study 6

\begin{tabular}{llccccc}
\hline DV & Predictor & Estimate & SE & Lower & Upper & N_Subj \\
\hline Sup. Eff. & Intercept & 5.21 & 0.10 & 5.02 & 5.39 & 101 \\
Sup. Eff. & PR & 0.65 & 0.08 & 0.51 & 0.80 & 101 \\
\hline Negative Mood & Intercept & 2.63 & 0.11 & 2.42 & 2.84 & 101 \\
Negative Mood & PR & -0.18 & 0.09 & -0.36 & 0.001 & 101 \\
Negative Mood & Pre Neg. Mood & 0.48 & 0.06 & 0.37 & 0.59 & 101 \\
\hline Positive Mood & Intercept & 3.89 & 0.08 & 3.73 & 4.05 & 101 \\
Positive Mood & PR & 0.30 & 0.07 & 0.16 & 0.45 & 101 \\
Positive Mood & Pre Pos. Mood & 0.61 & 0.06 & 0.48 & 0.73 & 101 \\
\hline IOS & Intercept & 4.96 & 0.10 & 4.76 & 5.16 & 101 \\
IOS & PR & 0.67 & 0.09 & 0.50 & 0.83 & 101 \\
\hline Closeness & Intercept & 6.41 & 0.06 & 6.29 & 6.52 & 101 \\
Closeness & PR & 0.43 & 0.05 & 0.33 & 0.52 & 101 \\
Closeness & Pre Closeness & 0.28 & 0.07 & 0.14 & 0.42 & 101 \\
\hline
\end{tabular}




\section{Table S38}

Effects of RES, Study 7

\begin{tabular}{llcccccc}
\hline DV & Predictor & Estimate & SE & Lower & Upper & Post_Prob N_Subj \\
\hline Support Effectiveness & Intercept & 5.34 & 0.08 & 5.19 & 5.50 & - & 110 \\
Support Effectiveness & RES & 0.85 & 0.07 & 0.70 & 0.99 & 1.00 & 110 \\
\hline Negative Mood & Intercept & 2.32 & 0.05 & 2.23 & 2.41 & - & 105 \\
Negative Mood & RES & -0.10 & 0.04 & -0.18 & -0.02 & 0.99 & 105 \\
Negative Mood & Pre Neg. Mood & 0.69 & 0.05 & 0.60 & 0.79 & - & 105 \\
\hline Positive Mood & Intercept & 4.17 & 0.07 & 4.03 & 4.32 & - & 105 \\
Positive Mood & RES & 0.02 & 0.07 & -0.11 & 0.15 & 0.60 & 105 \\
Positive Mood & Pre Pos. Mood & 0.93 & 0.07 & 0.80 & 1.06 & - & 105 \\
\hline IOS & Intercept & 5.04 & 0.12 & 4.80 & 5.27 & - & 107 \\
IOS & RES & 0.55 & 0.11 & 0.34 & 0.77 & 1.00 & 107 \\
\hline Closeness & Intercept & 5.72 & 0.07 & 5.57 & 5.86 & - & 110 \\
Closeness & RES & 0.34 & 0.07 & 0.21 & 0.47 & 1.00 & 110 \\
Closeness & Pre Closeness & 0.55 & 0.06 & 0.44 & 0.66 & - & 110 \\
\hline Change in Motivation & Intercept & 0.36 & 0.12 & 0.14 & 0.59 & - & 105 \\
Change in Motivation & RES & 0.26 & 0.10 & 0.06 & 0.47 & 0.99 & 105 \\
Change in Motivation & Pre Motivation & -0.43 & 0.09 & -0.60 & -0.26 & - & 105 \\
\hline Help with Speech & Intercept & 4.52 & 0.12 & 4.29 & 4.74 & - & 110 \\
Help with Speech & RES & 0.69 & 0.11 & 0.48 & 0.91 & 1.00 & 110 \\
\hline Speech Performance & Intercept & 3.87 & 0.07 & 3.73 & 4.01 & - & 106 \\
Speech Performance & RES & -0.03 & 0.07 & -0.16 & 0.10 & 0.32 & 106 \\
\hline
\end{tabular}


Table S39

Effects of PR, Study 7

\begin{tabular}{llcccccc}
\hline DV & Predictor & Estimate & SE & Lower & Upper & Post_Prob & N_Subj \\
\hline Support Effectiveness & Intercept & 5.34 & 0.10 & 5.15 & 5.53 & - & 110 \\
Support Effectiveness & PR & 0.71 & 0.10 & 0.52 & 0.90 & 1.00 & 110 \\
\hline Negative Mood & Intercept & 2.32 & 0.05 & 2.23 & 2.41 & - & 105 \\
Negative Mood & PR & -0.09 & 0.05 & -0.19 & 0.008 & 0.96 & 105 \\
Negative Mood & Pre Neg. Mood & 0.68 & 0.05 & 0.58 & 0.77 & - & 105 \\
\hline Positive Mood & Intercept & 4.17 & 0.07 & 4.03 & 4.31 & - & 105 \\
Positive Mood & PR & 0.04 & 0.07 & -0.10 & 0.18 & 0.69 & 105 \\
Positive Mood & Pre Pos. Mood & 0.93 & 0.07 & 0.79 & 1.06 & - & 105 \\
\hline IOS & Intercept & 5.04 & 0.11 & 4.83 & 5.26 & - & 107 \\
IOS & PR & 0.69 & 0.11 & 0.46 & 0.90 & 1.00 & 107 \\
\hline Closeness & Intercept & 5.72 & 0.07 & 5.58 & 5.86 & - & 110 \\
Closeness & PR & 0.39 & 0.08 & 0.24 & 0.54 & 1.00 & 110 \\
Closeness & Pre Closeness & 0.51 & 0.06 & 0.39 & 0.63 & - & 110 \\
\hline Change in Motivation & Intercept & 0.36 & 0.12 & 0.11 & 0.60 & - & 105 \\
Change in Motivation & PR & 0.05 & 0.11 & -0.17 & 0.27 & 0.65 & 105 \\
Change in Motivation & Pre Motivation & -0.41 & 0.09 & -0.58 & -0.24 & - & 105 \\
\hline Help with Speech & Intercept & 4.52 & 0.13 & 4.26 & 4.77 & - & 110 \\
Help with Speech & PR & 0.46 & 0.13 & 0.22 & 0.72 & 1.00 & 110 \\
\hline Speech Performance & Intercept & 3.87 & 0.07 & 3.73 & 4.01 & - & 106 \\
Speech Performance & PR & -0.10 & 0.07 & -0.24 & 0.03 & 0.07 & 106 \\
\hline
\end{tabular}




\section{Table S40}

Fixed Effects of RES on cardiovascular responses, Study 7

\begin{tabular}{lcccccccc}
\hline Coefficient & Estimate & SE & Lower & Upper & Post_Prob & N_Subj & N_Obs \\
\hline Intercept & 670.12 & 11.43 & 646.52 & 692.56 & - & 106 & 3226 \\
RES & -0.06 & 9.86 & -19.20 & 19.43 & 0.50 & 106 & 3226 \\
Baseline vs. Speech & 135.89 & 10.60 & 116.15 & 157.02 & 1.00 & 106 & 3226 \\
Support vs. Speech & 58.14 & 6.33 & 45.97 & 70.76 & 1.00 & 106 & 3226 \\
Recovery vs. Speech & 137.66 & 8.65 & 121.04 & 155.04 & 1.00 & 106 & 3226 \\
RES x Support vs. Speech & -6.69 & 5.85 & -17.77 & 5.05 & 0.13 & 106 & 3226 \\
RES x Recovery vs. Speech & -6.73 & 8.04 & -22.32 & 9.27 & 0.20 & 106 & 3226 \\
RES x Baseline vs. Speech & -6.31 & 9.54 & -24.73 & 12.29 & 0.25 & 106 & 3226 \\
\hline
\end{tabular}

\section{Table S41}

Fixed Effects of PR on cardiovascular responses, Study 7

\begin{tabular}{lccccccc}
\hline Coefficient & Estimate & SE & Lower & Upper & Post_Prob & N_Subj & N_Obs \\
\hline Intercept & 671.01 & 10.82 & 649.84 & 691.65 & - & 106 & 3226 \\
PR & -11.78 & 11.00 & -32.72 & 10.68 & 0.14 & 106 & 3226 \\
Baseline vs. Speech & 135.09 & 10.54 & 113.96 & 155.76 & 1.00 & 106 & 3226 \\
Support vs. Speech & 57.54 & 6.42 & 45.18 & 70.21 & 1.00 & 106 & 3226 \\
Recovery vs. Speech & 136.45 & 8.68 & 118.61 & 153.42 & 1.00 & 106 & 3226 \\
PR x Baseline vs. Speech & -3.13 & 10.07 & -22.58 & 16.78 & 0.62 & 106 & 3226 \\
PR x Support vs. Speech & 0.74 & 6.22 & -11.85 & 12.63 & 0.45 & 106 & 3226 \\
PR x Recovery vs. Speech & -1.36 & 8.20 & -17.63 & 14.60 & 0.57 & 106 & 3226 \\
\hline
\end{tabular}




\section{Table S42}

Random Effects for model predicting cardiovascular responses, RES, Study 7

\begin{tabular}{lcccc}
\hline Term & Estimate & SE & Lower & Upper \\
\hline Intercept SD & 112.39 & 8.35 & 97.74 & 130.01 \\
Baseline vs. Speech SD & 99.88 & 7.62 & 86.02 & 115.85 \\
Support vs. Speech SD & 56.71 & 5.03 & 47.50 & 67.41 \\
Recovery vs. Speech SD & 79.04 & 6.56 & 67.03 & 92.68 \\
Intercept-Baseline vs. Speech Cor & -0.27 & 0.10 & -0.45 & -0.06 \\
Intercept-Support vs, Speech Cor & -0.22 & 0.11 & -0.42 & 0.002 \\
Intercept-Recovery vs. Speech Cor & -0.19 & 0.11 & -0.39 & 0.04 \\
Support vs. Speech-Baseline vs. Speech Cor & 0.67 & 0.07 & 0.53 & 0.78 \\
Support vs. Speech-Recovery vs. Speech Cor & 0.62 & 0.08 & 0.45 & 0.75 \\
Recovery vs. Speech-Baseline vs. Speech Cor & 0.81 & 0.04 & 0.71 & 0.88 \\
Residual & 39.67 & 0.56 & 38.58 & 40.80 \\
AR(1) & 0.39 & 0.03 & 0.34 & 0.45 \\
\hline
\end{tabular}

\section{Table S43}

Random Effects for model predicting cardiovascular responses, PR, Study 7

\begin{tabular}{lcccc}
\hline Term & Estimate & SE & Lower & Upper \\
\hline Intercept SD & 111.44 & 8.53 & 96.18 & 129.61 \\
Baseline vs. Speech SD & 100.49 & 7.73 & 86.36 & 116.87 \\
Support vs. Speech SD & 57.49 & 4.96 & 48.48 & 67.87 \\
Recovery vs. Speech SD & 79.47 & 6.62 & 67.33 & 93.98 \\
Intercept-Baseline vs. Speech Cor & -0.27 & 0.10 & -0.45 & -0.08 \\
Intercept-Support vs, Speech Cor & -0.22 & 0.11 & -0.41 & 0.004 \\
Intercept-Recovery vs. Speech Cor & -0.18 & 0.11 & -0.38 & 0.03 \\
Support vs. Speech-Baseline vs. Speech Cor & 0.68 & 0.06 & 0.54 & 0.79 \\
Support vs. Speech-Recovery vs. Speech Cor & 0.63 & 0.08 & 0.46 & 0.76 \\
Recovery vs. Speech-Baseline vs. Speech Cor & 0.81 & 0.04 & 0.72 & 0.88 \\
Residual & 39.68 & 0.57 & 38.60 & 40.82 \\
AR(1) & 0.39 & 0.03 & 0.34 & 0.45 \\
\hline
\end{tabular}




\section{References}

Bolger, N., Zee, K. S., Rossignac-Milon, M., \& Hassin, R. R. (2019). Causal processes in psychology are heterogeneous. Journal of Experimental Psychology: General, 148, 601-618.

Cavallo, J. V., Zee, K. S., \& Higgins, E. T. (2016). Giving the help that is needed: How Regulatory Mode impacts tailoring social support provision. Personality and Social Psychology Bulletin, 42, 1111-1128.

Zee, K. S., Cavallo, J. V., Flores, A. J., Bolger, N., \& Higgins, E. T. (2018). Motivation moderates the effects of social support visibility. Journal of Personality and Social Psychology, 114, 735-765. 